\title{
NONLINEAR DYNAMICS AND CHAOS OF THE DAISYWORLD EMPLOYED FOR GLOBAL WARMING DESCRIPTION
}

\author{
VIOLA, F.M. ${ }^{1}{ }^{*}-$ SAVI, M.A. ${ }^{1}-$ PAIVA, S.L.D. ${ }^{1}-$ BRASIL, A.C.P. JR. ${ }^{2}$ \\ ${ }^{I}$ Universidade Federal do Rio de Janeiro \\ COPPE - Department of Mechanical Engineering \\ 21.941.972 - Rio de Janeiro - RJ - Brazil, P.O. Box 68.503 \\ (e-mail:fmviola@gmail.com,supaiva@gmail.com, savi@mecanica.ufrj.br) \\ ${ }^{2}$ Universidade de Brasília, Department of Mechanical Engineering \\ 70.910.900 - Brasília - DF - Brazil \\ (e-mail:brasiljr@unb.br) \\ *Corresponding author \\ e-mail:fmviola@gmail.com \\ (Received 26 ${ }^{\text {th }}$ September 2012; accepted $2^{\text {nd }}$ September 2013)
}

\begin{abstract}
The mathematical modeling of ecological phenomena may describe time evolution and spatial distribution being capable to explain some important characteristics of ecological systems. Although there are many difficulties related to the system description, their modeling may define at least a system caricature, which may be useful for different goals. This contribution deals with the modeling of the global warming in a nonlinear dynamics point of view. Mathematical modeling is based on the daisyworld that is able to describe the global regulation that can emerge from the interaction between life and environment. In brief, daisyworld represents life by daisy populations while the environment is represented by temperature. Here, two daisy populations are of concern, black and white daisies, and an extra variable related to greenhouse gases is incorporated in the model allowing the analysis of the global warming. Moreover, transient analysis of temperature evolution is of concern. Climate variability is represented by a sinusoidal variation of the luminosity. Numerical simulations are investigated in order to present a qualitative description of the phenomenon. Daisyworld dynamics presents a rich behavior including chaos.
\end{abstract}

Keywords: Global warming, daisyworld, nonlinear dynamics, chaos, ecology.

\section{Introduction}

Global warming is a specific case of the more general term climate change. Although climate change can be related to either natural or anthropogenic causes, it is usually associated with human activities. In general, it is important to establish a difference between climate change and climate variability. Climate change is related to permanent changes while climate variability denotes deviations of climate conditions over a period of time due to natural phenomena (WMO, 2010).

The mechanism of the Earth's heating is related to the energy balance where the main aspects are the radiation energy from the sun (short waves) and the thermal radiation from the Earth that is radiated out to the space (long waves). The atmosphere plays an essential role in this process and the presence of greenhouse gases tends to change this balance since they are transparent to the sun short wave radiation, however, they absorb some of the longer infrared radiation emitted from the Earth. Therefore, the increase amounts of these gases in the atmosphere tend to cause the increase of Earth's temperature. In this regard, the primary cause of global warming is the release of 
greenhouse gases, leading to the increase of the so-called greenhouse effect (Houghton, 2005).

There are numerous modeling efforts trying to analyze either climate change or climate variability and their effects on the Earth. In general, one can establish the following classification (Alexiadis, 2007); general circulation models (GCMs); modelbased methods (MBMs) or empirical models; planet's dynamics models (PDMs). Moreover, we can highlight the existence of models built upon time series analysis (Viola et al., 2010). GCMs consider physical aspects of system dynamics including conservation of physical variables. MBMs use some empirical observations and/or statistical tools from experimental time series and therefore, do not deal with system's physics directly. PDM are based on a simplified description of the system dynamics and falls between the previous two categories. Time series analysis tries to build a model from experimental data.

The mathematical modeling of ecological phenomena has an increasing importance in recent years (Jorgensen, 1999; Savi, 2005, 2006). These models may describe time evolution and spatial distribution being able to explain some important characteristics of ecological systems. The mathematical analysis is exploiting the possibility that many of these phenomena may have their roots in some underlying dynamical effect. Although there are many difficulties related to the system description, their modeling may define at least a system caricature, which may be useful for different goals.

Gaia theory of the Earth establishes the self-regulation of the planetary system being originally proposed by James Lovelock, in 1972. The daisyworld is an archetypal model of the Gaia theory representing a mathematical description of this idea (Watson \& Lovelock, 1983). This model is able to describe the global regulation that can emerge from the interaction between life and environment. Daisyworld represents life by daisy populations while the environment is represented by temperature (Lovelock, 1992; Lenton \& Lovelock, 2000, 2001). Wood (2008) presented a general overview about the literature associated with daisyworld emphasizing its main characteristics and different approaches for its analysis.

Darwinian characteristics of the daisyworld were investigated in different references. Robertson \& Robinson (1998) analyzed the changes in optimal temperature of the daisy populations by considering that environmental conditions are unchanged. Lenton \& Lovelock (2000) incorporated the environmental changes in the analysis. Sugimoto (2002) discussed mathematical solutions of these two daisyworld models. Cohen \& Rich (2000) treated some daisy competition aspects in the daisyworld, evaluating the temperature changes. Ackland et al. (2003) discussed catastrophic alterations of the daisyworld. Spatiotemporal aspects of the daisyworld were treated in some references as Adams et al. (2003) that investigated a one-dimensional model of the daisyworld evaluating heat exchanges.

One of the main characteristics of the daisyworld is the capability to describe either local or global phenomena. Local analysis can be done by considering the solar luminosity of some part of the planet, representing its general evolution through time. On the other hand, global analysis represents an average behavior of the whole planet. In this regard, time scale may be related from months to thousands of years. Therefore, it is possible to treat either season variations through year or climate variability through millenniums. Staley (2002) and Charlson et al. (1987) discussed the differences between the local and global effects in which the global effect is essentially related to 
local effects. Wood (2008) established that daisyworld contains a fixed relationship between local benefit and global regulation.

Wood et al. (2010) treated daisy and trees in the daisyworld. Both populations have independent growth in the same environment. Salazar \& Poveda (2009) incorporated clouds and hydrological cycle in the regulation of the daisyworld.

Concerning the dynamical behavior of the daisyworld, Zeng et al. (1990) presents an investigation about chaos considering a discrete version. Nevertheless, the proposed discrete version is actually, different from the continuous daisyworld model, as pointed by Jascourt \& Raymond (1992). Wood (2008) discussed some aspects of the chaos in the daisyworld, showing some simulations reported in literature, pointing that it is a controversial subject.

This contribution deals with the modeling of the global warming in a nonlinear dynamic point of view. We are essentially interested in a qualitative description of the phenomenon. Mathematical model is based on the classical daisyworld incorporating an extra variable related to greenhouse gases. In brief, daisyworld represents life by two daisy populations (black and white) while the environment is represented by temperature. Besides, energy equation is considered in order to investigate transients phenomena related to temperature variation. Climate variability is represented by a sinusoidal variation of the luminosity. Numerical simulations are carried out in order to present a qualitative description of the global warming. Daisyworld presents a rich dynamical behavior and this paper is particularly interested in complex responses. In this regard, this contribution has two main goals related to a qualitatively investigation of the daisyworld: to incorporate greenhouse gases in the analysis; to investigate the possibility of chaotic behavior.

This article is organized as follows: after this introduction, a discussion about the daisyworld mathematical model is presented. Then, numerical simulation of the classical daisyworld is performed. Afterward, the effect of greenhouse gases is investigated. The influence of climate variability is then analyzed. Under this condition, a complex dynamical behavior emerges and a deep investigation is carried out. Basically, we are especially interested in the chaotic behavior of the daisyworld and therefore, we present an investigation involving nonlinear tools as bifurcation diagrams and Lyapunov exponents. Finally, concluding remarks are presented.

\section{Daisyworld model}

Climate system has an inherent complexity due to different kinds of phenomena involved. The equilibrium of this system is a consequence of different aspects related to the atmosphere, oceans, biosphere and many others, and the sun activity provides the driving force of the system. The Earth's heating mechanism may be understood as the balance between the radiation energy from the sun and the thermal radiation from the Earth and the atmosphere that is radiated out to the space. The presence of greenhouse gases tends to change this balance since they are transparent to the sun short wave radiation, however, they absorb some of the longer infrared radiation emitted from the Earth. Therefore, the increase amounts of these gases make the Earth cool more difficult increasing the Earth's surface temperature.

Watson \& Lovelock (1983) proposed a model to demonstrate that global regulation can emerge from the interaction between life and environment. This archetypal model was called daisyworld representing an imaginary planet populated by organisms in coexistence. The daisyworld is basically composed of the environment, represented by 
the temperature, and of daisy populations, representing life. The original daisyworld includes only two populations of daisies but further investigations include herbivores and carnivores as well as colored daisies (Lovelock, 1992).

The first step of the daisyworld modeling is the definition of biotic components of dynamical system, represented by daisies, which evolution is described by the following general equation where $\alpha_{i}(i=1,2, \ldots N)$ represents the area coverage by daisy populations:

$$
\dot{\alpha}_{i}=\alpha_{i}\left\lfloor\alpha_{g} \beta\left(T_{i}\right)-\gamma\right\rfloor
$$

where dot represents time derivative, $\beta=\beta\left(T_{i}\right)$ represents the growth rate that is temperature dependent and $\gamma$ is the death rate. Daisy colors define the amount of energy absorption and the balance between daisy populations controls the planet temperature. A first approach to this archetypal model is to consider only two daisy populations: black, $\alpha_{b}$, and white, $\alpha_{w}$. Black daisies absorb more energy while white daisies absorb less energy.

The functional form for $\beta_{i}$ is usually assumed to be a symmetric single-peaked function as follows:

$$
\beta_{i}(T)=\left\{\begin{array}{cc}
B\left[1-\left(\frac{T_{\mathrm{opt}}-T_{i}}{k}\right)^{2}\right] & \quad\left|T_{\mathrm{opt}}-T_{i}\right|<k \\
0 & \text { otherwise }
\end{array}\right.
$$

where $T_{\text {opt }}$ is the optimal temperature, usually considered as $T_{\mathrm{opt}}=295 \mathrm{~K}=22.5{ }^{\circ} \mathrm{C}$. The parabolic width $k$ is chosen in order to establish proper life conditions as for example, between $5^{\circ} \mathrm{C}$ and $40^{\circ} \mathrm{C}$ (De Gregorio et al., 1992), which is related to $k=17.5$. In the same way, $B$ alters these values in order to represent different environmental characteristics.

The greenhouse effect can be understood as a deviation from the blackbody radiation in the equation of the planetary temperature (Nevison et al., 1999). Here, the effect of greenhouse gases is incorporated in the daisyworld considering a new state variable that affects global dynamics in two distinct forms: increasing the albedo and land occupation. In this regard, variable $G$ limits the life of the daisy populations and affects the daisyworld's albedo in a similar way of the black daisies. Under this assumption, it is possible to define this variable as a known time series related to greenhouse gases:

$$
G=G(t)
$$

This time series can be measured, being the consequence of the balance between the emission of gases and the nature absorption.

The variable $\alpha_{g}$ is the fractional area coverage of the planet represented by:

$$
\alpha_{g}=p-\sum_{i=1}^{N} \alpha_{i}-G
$$

Here, $p$ represents the proportion of land suitable for the growth of daisies and $N$ represents the biodiversity related to the number of populations involved in the system. 
The mean planetary albedo of the daisyworld, $A$, can be estimated from the individual albedo of each population ( $a_{i}$ for daisies, $a_{g}$ for the bare ground and $a_{G}$ due to greenhouse gases effect):

$$
A=\alpha_{g} a_{g}+\sum_{i=1}^{N} \alpha_{i} a_{i}+G a_{G}
$$

Afterwards, the local temperature of each population is defined as follows:

$$
\begin{aligned}
& T_{i}^{4}=q\left(A-a_{i}\right)+T^{4} \\
& T_{g}^{4}=q\left(A-a_{g}\right)+T^{4} \\
& T_{G}^{4}=q\left(A-a_{G}\right)+T^{4}
\end{aligned}
$$

where $T$ is the globally-averaged temperature of the daisyworld, and $q$ is a constant used to calculate local temperature as a function of albedo (Watson \& Lovelock, 1983). Finally, it is important to establish the thermal balance of the daisyworld (Foong, 2006), and therefore, the absorbed energy is given by (Nevison et al., 1999):

$$
\dot{T}=\frac{1}{c}\left[S L(1-A)-\sigma T^{4}\right]
$$

$L$ is the solar luminosity and $S$ is the solar constant that establishes the average solar energy, $S L ; \sigma$ is the Stefan-Boltzmann constant; $c$ is a measure of the average heat capacity or thermal inertia of the planet. This equation induces oscillations either in temperature or daisy populations. Therefore, the definition of the value of the constant is responsible for the kind of response defining the amplitude and frequency of these oscillations (Nevison et al., 1999).

A key difference between climate change and climate variability is the persistence of anomalous conditions. In order to investigate the effect of climate variability in the daisyworld, it is assumed a solar luminosity with a sinusoidal variation represented as follows:

$$
L=\bar{L}+L_{0} \sin (\omega t)
$$

Note that $\bar{L}=\bar{L}(t)$ and the term $L_{0} \sin (\omega t)$ represents a perturbation that is associated with climate variability.

The daisyworld model can be simulated using classical procedures for numerical integration. Here, the fourth order Runge-Kutta method is employed. In general, the following parameters are assumed for numerical simulations: $q=2.06 \times 10^{9} \mathrm{~K}^{4}, \sigma=$ $5.67 \times 10^{-8} \mathrm{~W} / \mathrm{m}^{2} \mathrm{~K}^{4}, S=917 \mathrm{~W} / \mathrm{m}^{2}$. Other parameters are varied in order to analyze different system conditions. Moreover, it is important to highlight that only black and white daisy populations are considered, which means that $N=2$. 


\section{Classical dailyworld}

This section investigates the dynamics of the daisyworld in order to establish a proper comprehension of the planetary self-regulation. Classical daisyworld is of concern and therefore, greenhouse gases and climate variability are not treated in this section. Parameters used in this simulation are $a_{w}=0.75, a_{b}=0.25, a_{g}=0.5, a_{G}=0.25$, $G=0, \gamma=0.3, B=1$ and the initial conditions $\alpha_{w}=\alpha_{b}=0.01$. All simulation employs time steps smaller than 0.01 .

Initially, constant luminosity is of concern assuming the classical situation with $c=$ 0 . The daisyworld has self-regulation due to the interaction between life and environment, respectively represented by daisy populations and the daisyworld temperature. Therefore, the planetary system tends to maintain a constant temperature adequate for life due to the interaction between black and white daisy populations. The increase of the black daisies tends to increase the planet temperature since they absorb more energy, and the opposite occurs concerning white daisies. Hence, the population growth is in such a way that temperature remains constant in a favorable value as shown in Figure 1. This result is the classical result obtained in the earlier cited papers (Watson \& Lovelock, 1983; Lovelock, 1988).

Afterwards, the influence of thermal inertia is investigated. Basically, two different values of parameter $c$ are of concern: 300 and $3000 \mathrm{~J} / \mathrm{m}^{2} \mathrm{~K}$ s. Figures $2-3$ show the system response for these situations. Note that there is an oscillatory behavior from daisy populations that causes temperature oscillation. These oscillations occur around a mean value obtained when $c=0$ but may reach large amplitudes. In some cases, the minimum values of these oscillations reach critical levels near to the population extinction.
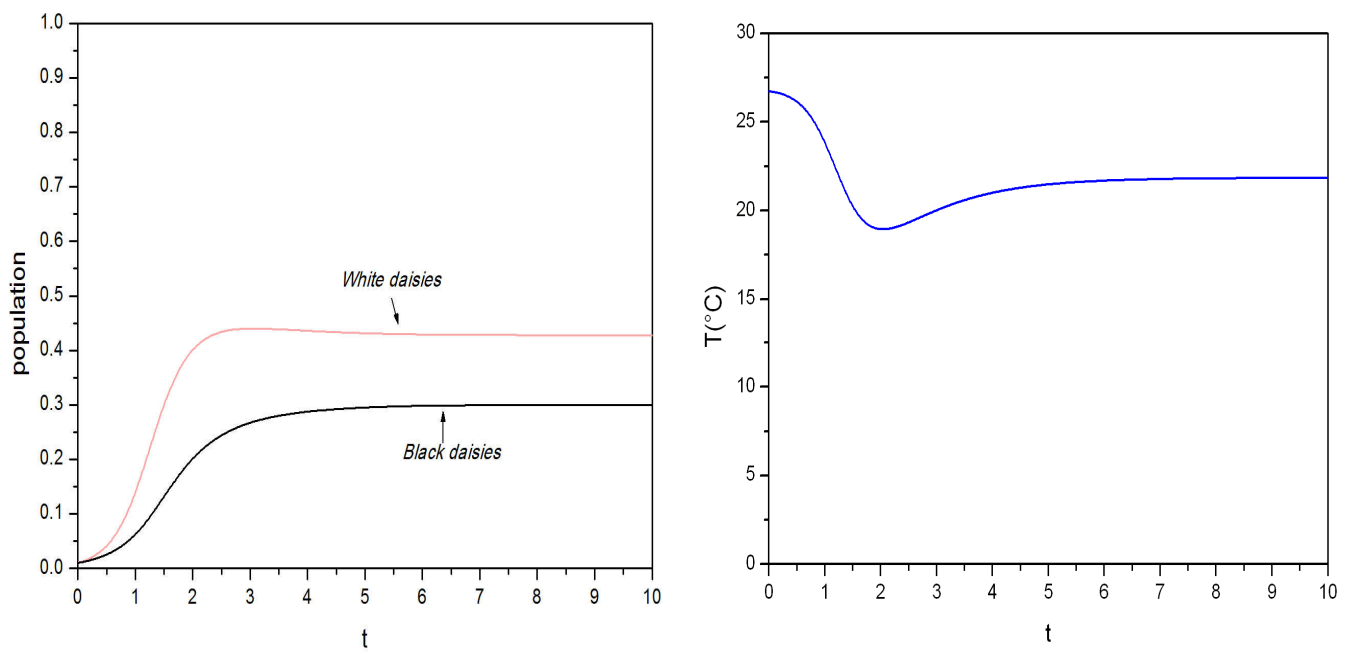

Figure 1. Daisyworld response with constant solar luminosity $\left(\bar{L}=1, L_{0}=0\right)$ and $c=0$. Daisy population (left) and the temperature (right) 

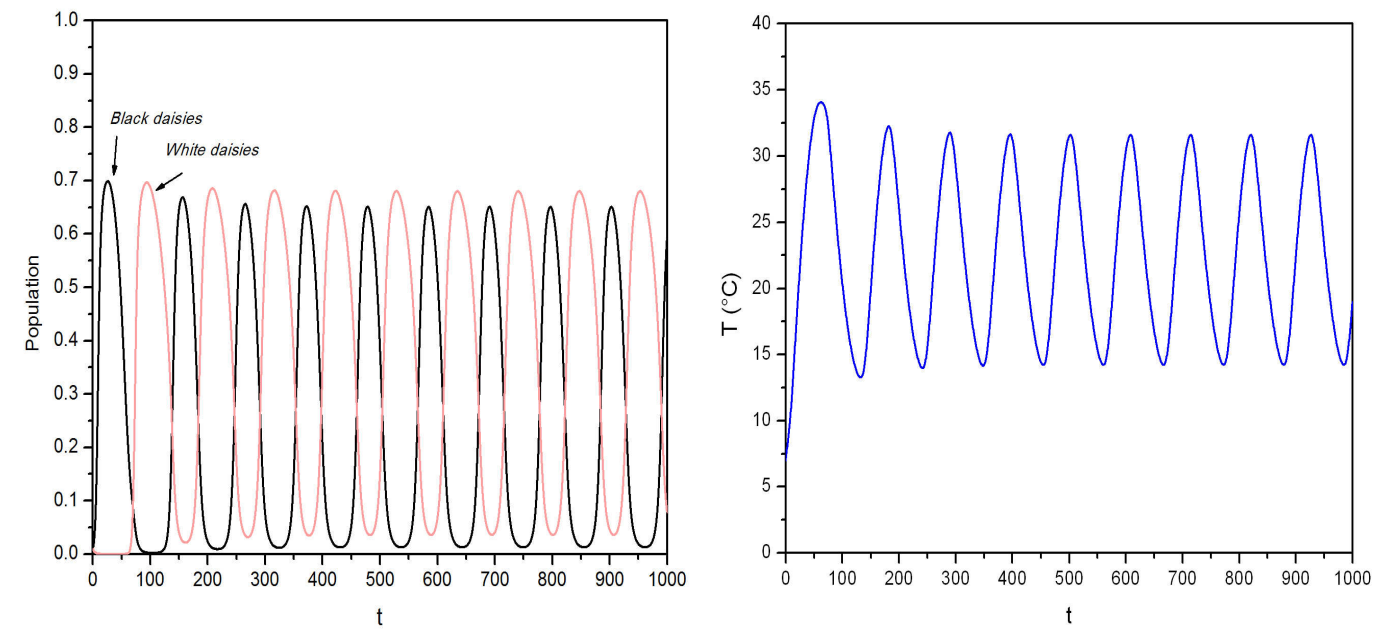

Figure 2. Daisyworld response with constant solar luminosity $\left(\bar{L}=1, L_{0}=0\right)$ and $c=300$ $\mathrm{J} / \mathrm{m}^{2} \mathrm{~K}$ s. Daisy population (left) and the temperature (right)
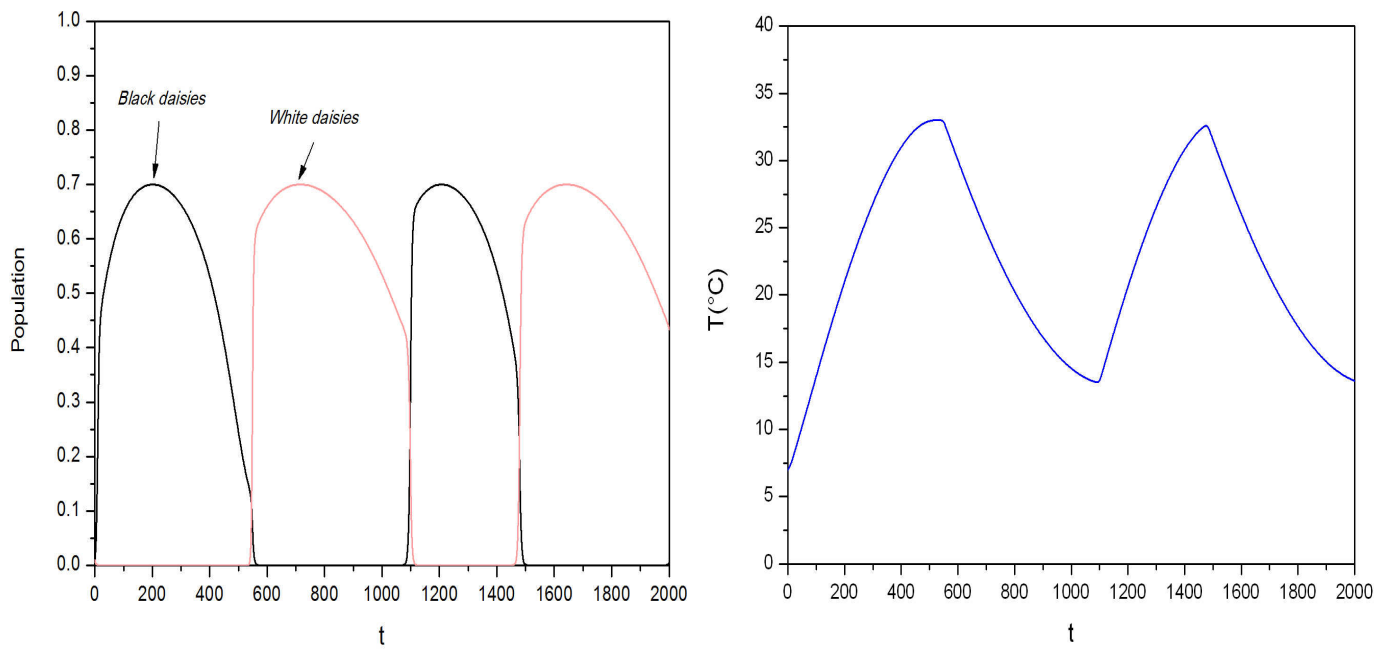

Figure 3. Daisyworld response with constant solar luminosity $\left(\bar{L}=1, L_{0}=0\right)$ and $c=3000$ $\mathrm{J} / \mathrm{m}^{2} \mathrm{~K}$ s. Daisy population (left) and the temperature (right)

A linear increase of the solar luminosity $(0.75 \leq \bar{L} \leq 1.7$; L0 $=0)$ is now in focus representing a more realistic representation of the solar activity. Under this condition, the daisyworld temperature would tend to increase linearly, following the luminosity increase. Nevertheless, the self-regulation of the daisyworld tends to maintain a constant temperature due to the interaction between black and white daisy populations. Once again, the increase of the black daisies tends to increase the planet temperature since they absorb more energy. This occurs when the solar luminosity has small values. The increase in solar luminosity causes the decrease of the black daisies population and the increase of the white daises. This balance is represented by a tendency of constant values of temperature. Figure 4 shows the evolution of the daisy populations and the temperature for $\mathrm{c}=0$. It is clear that, when the luminosity has small values, black daisies are preponderant. The more luminosity increases, the more white daisies 
increase. Figure $4 b$ also establishes a comparison between temperature evolution for dead planet (without life, or daisies) and the planet with life (with daisies).
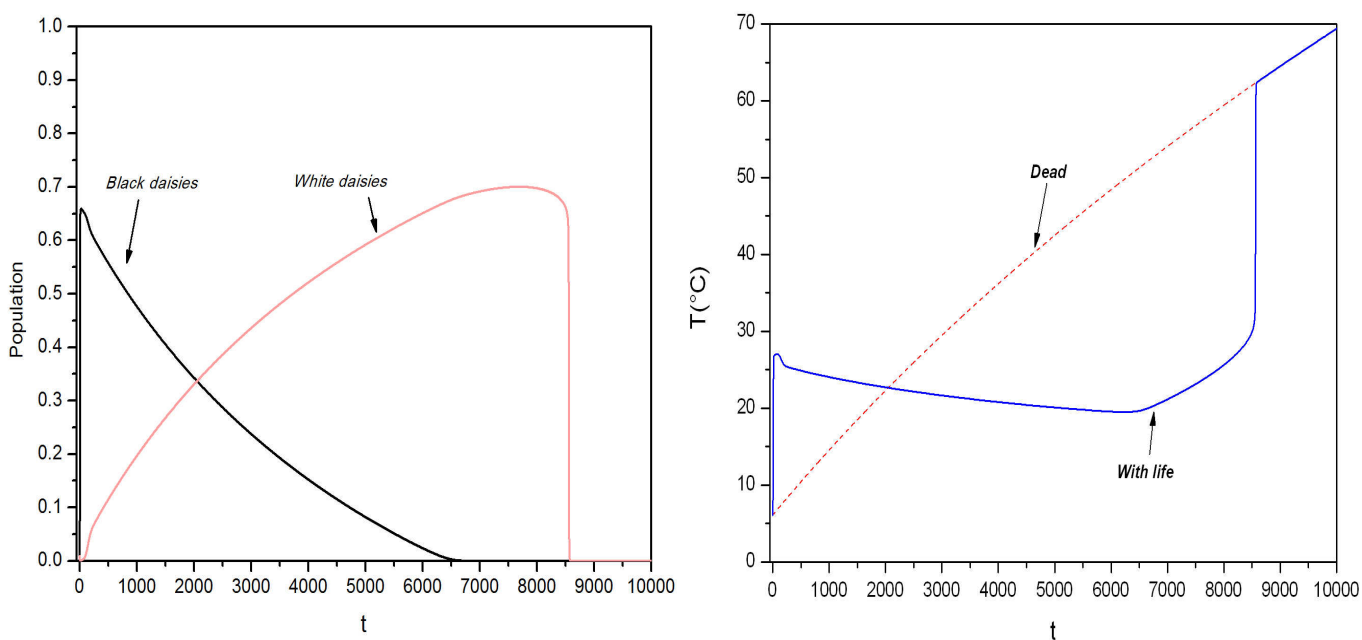

Figure 4. Daisyworld response with linear increase solar luminosity $\left(0.75 \leq \bar{L} \leq 1.7 ; L_{0}=0\right)$ and $c=0$

The influence of the planet thermal inertia is now in focus by assuming two different values of parameter c: 300 (Figure 5) and 3000 (Figure 6) $\mathrm{J} / \mathrm{m}^{2} \mathrm{~K}$ s. Once again, the increase of the thermal inertia tends to promote oscillatory variations of all involved variables and it should be highlighted a proper balance between both populations and the temperature.
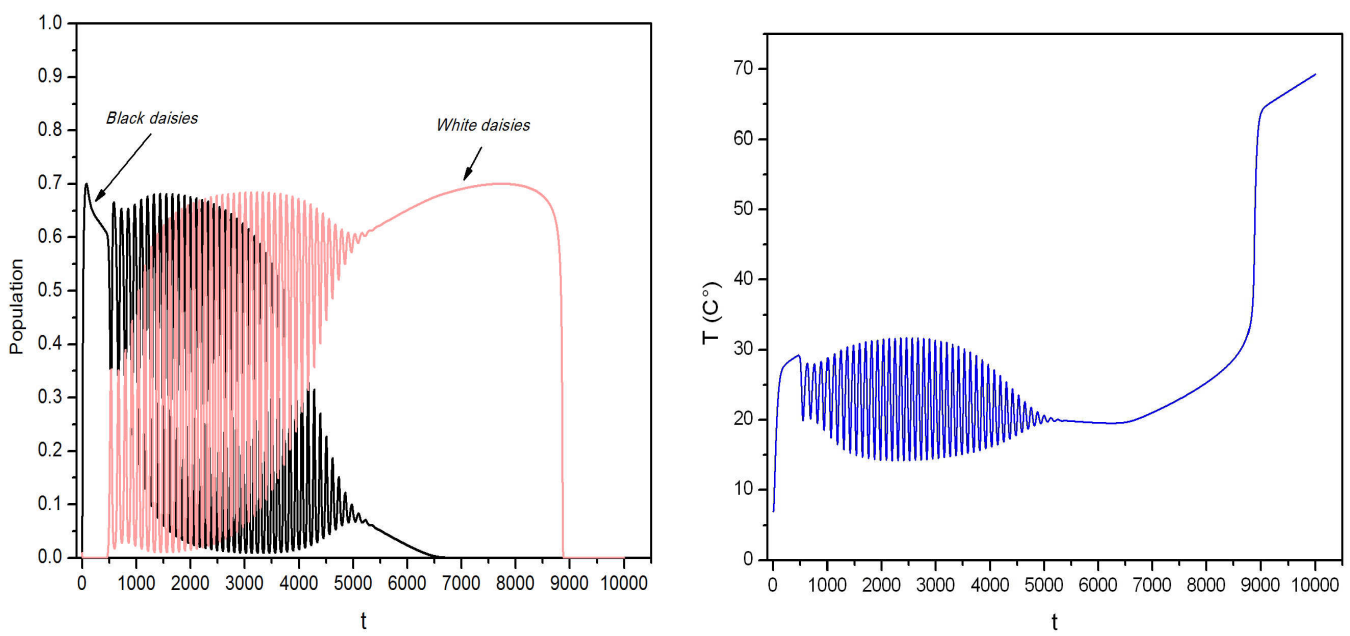

Figure 5. Daisyworld response with linear increase solar luminosity $\left(0.75 \leq \bar{L} \leq 1.7 ; L_{0}=0\right)$ and $c=300 \mathrm{~J} / \mathrm{m}^{2} \mathrm{~K}$ s. Daisy population (left) and the temperature (right) 

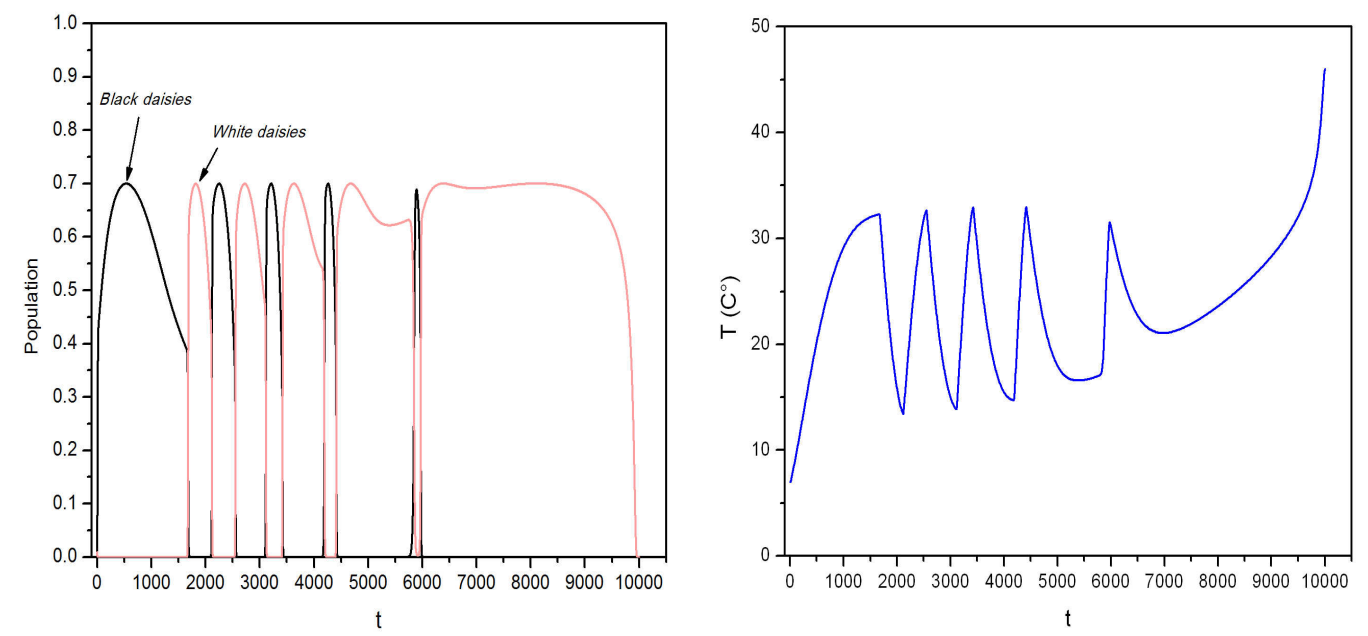

Figure 6. Daisyworld response with linear increase solar luminosity $\left(0.75 \leq \bar{L} \leq 1.7 ; L_{0}=0\right)$ and $c=3000 \mathrm{~J} / \mathrm{m}^{2} \mathrm{~K}$ s. Daisy population (left) and the temperature (right)

\section{Daisyworld with greenhouse gases}

This section discusses the effect of greenhouse gases in the daisyworld dynamics. Basically, it is assumed that these gases are known being related to a time series. Experimental values are used as a reference to characterize the general tendency of these gases. In this regard, $\mathrm{CO}_{2}$ concentration from 1980 to 2010 is used to estimate this general tendency (NOAA, 2011) and Figure 7 presents the average of the annual concentration and the $G$ function. Both curves present a linear increase with the same slope, establishing the same qualitative behavior. Numerical simulations are performed assuming time steps smaller than 0.01. The basic idea of this section is to establish a comparison with results of the preceding section that do not consider greenhouse gases.
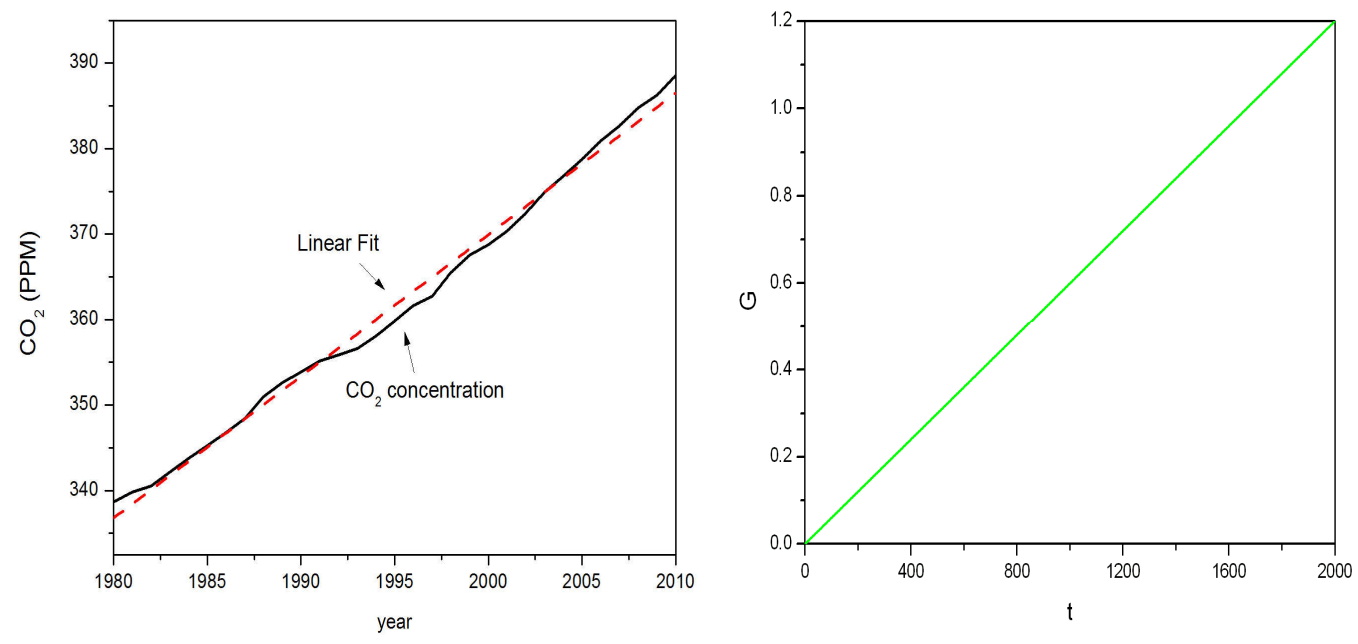

Figure 7. Annual average of the $\mathrm{CO}_{2}$ concentration based on NOAA (2011)

Initially, luminosity with linear increase is treated $\left(0.75 \leq \bar{L} \leq 1.7 ; L_{0}=0\right)$. Parameters used in this simulation are the same from the previous section, except for the 
greenhouse gases variable $G$. Figure 8 presents results of the daisyworld response showing the same behavior of the previous case, without greenhouse gases. It is important to observe that the increase of the daisyworld temperature promoted by greenhouse gases tends to cause an earlier death of daisy populations when compared to the planet without gases. This Figure also presents a comparison between the dead and the live planets showing how life interaction promotes the self-regulation of the planet.
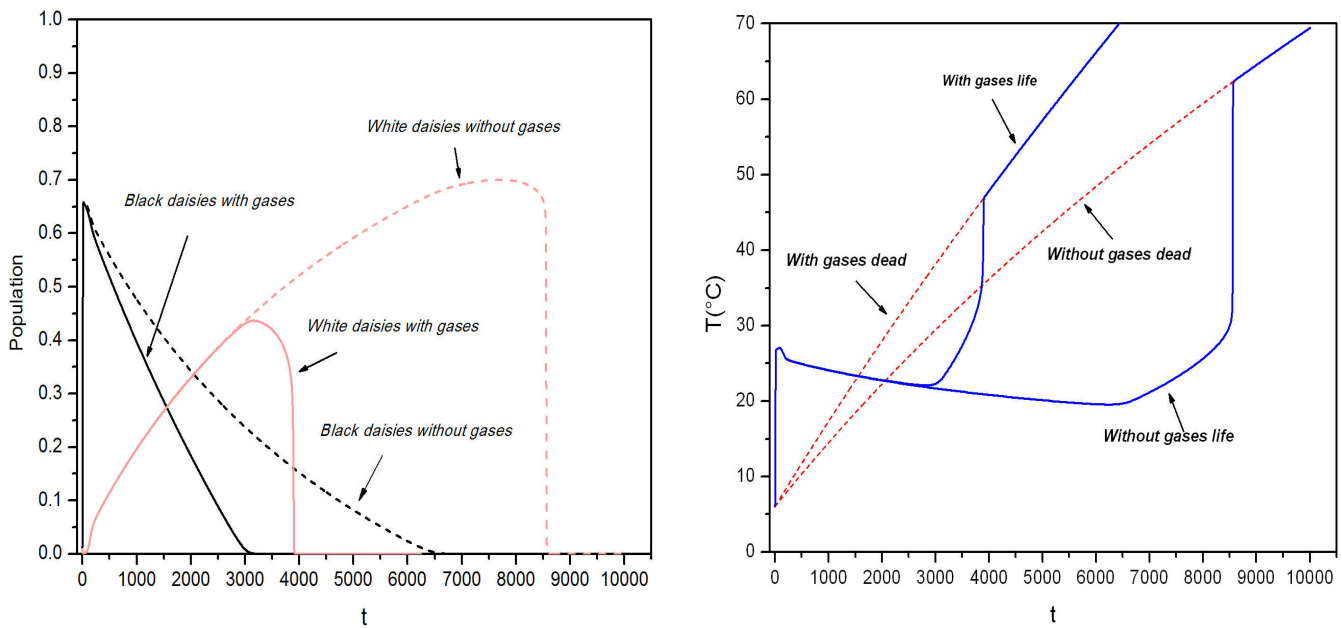

Figure 8. Daisyworld response with linear increase solar luminosity $\left(0.75 \leq \bar{L} \leq 1.7 ; L_{0}=0\right)$, greenhouse gases variable $(0.0 \leq G \leq 0.8)$ and $c=0$

The forthcoming analysis considers the influence of the planet thermal inertia by assuming linear increase of the solar luminosity $\left(0.75 \leq \bar{L} \leq 1.7 ; L_{0}=0\right)$. Figures $9-10$ show the system behavior for different values of parameter $c: 300$ (Figure 9) and 3000 (Figure 10) $\mathrm{J} / \mathrm{m}^{2} \mathrm{~K}$ s. Once again, note that there is a proper balance between both populations and the thermal inertia tends to cause an oscillatory response around the constant equilibrium temperature.
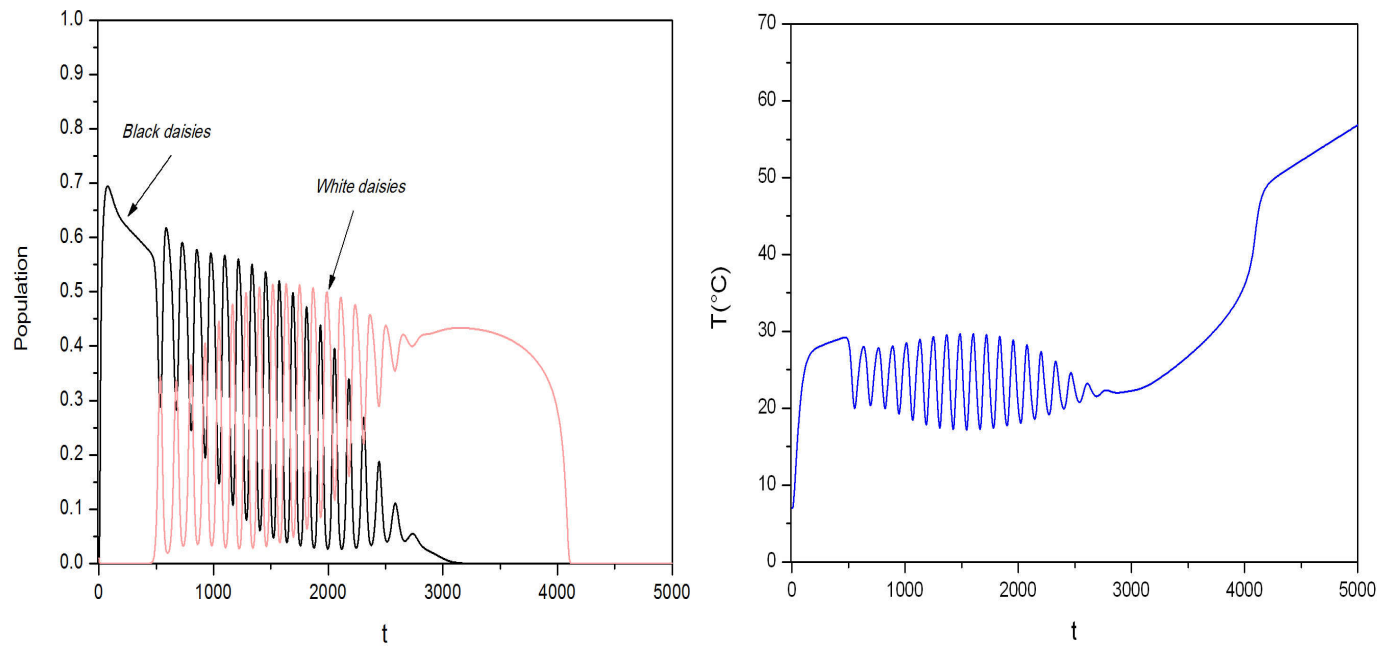

Figure 9. Daisyworld response with linear increase solar luminosity $\left(0.75 \leq \bar{L} \leq 1.7 ; L_{0}=0\right)$, greenhouse gases variable $(0.0 \leq G \leq 0.8)$ and $c=300 \mathrm{~J} / \mathrm{m}^{2} \mathrm{Ks}$ 

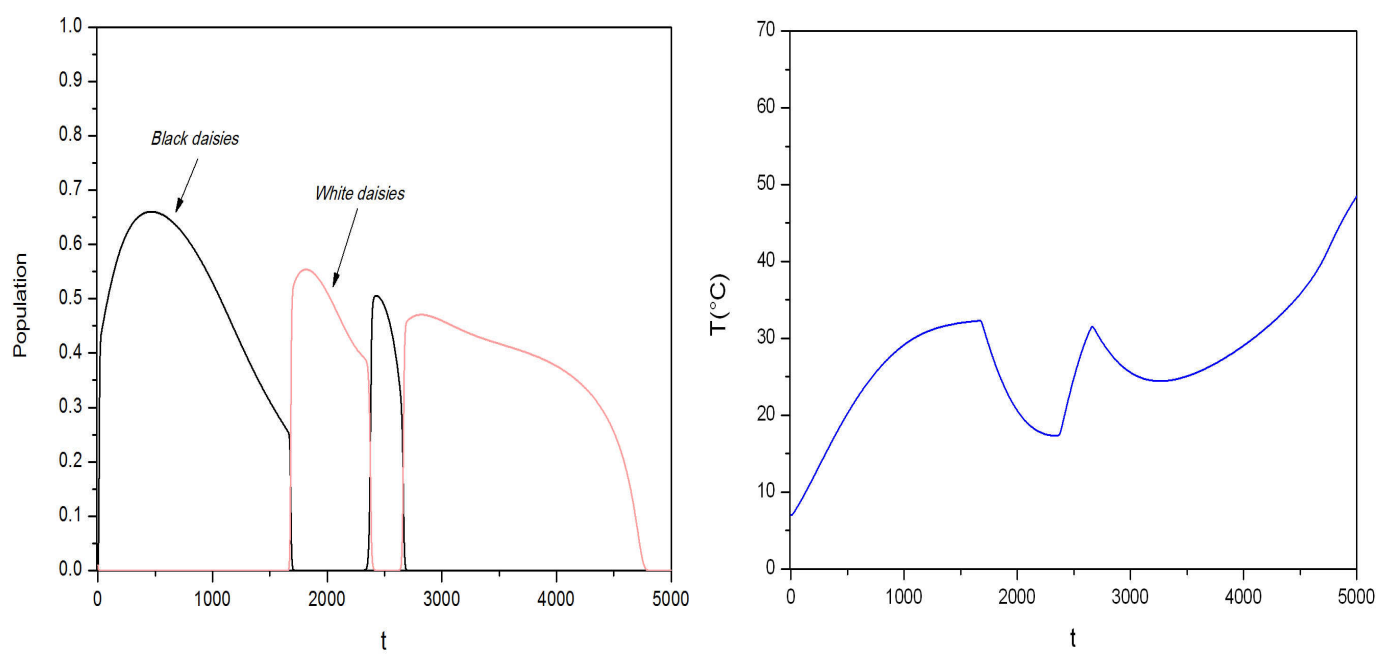

Figure 10. Daisyworld response with linear increase solar luminosity $\left(0.75 \leq \bar{L} \leq 1.7 ; L_{0}=0\right)$, greenhouse gases variable $(0.0 \leq G \leq 0.8)$ and $c=3000 \mathrm{~J} / \mathrm{m}^{2} \mathrm{Ks}$

\section{Daisyworld and climate variability}

The climate variability is now concerned by assuming a linear increase of luminosity $(0.75 \leq \bar{L} \leq 1.7)$ and a sinusoidal variability with $L_{0}=0.1$ and $\omega=0.01$, as presented in Figure 11. Besides, it is assumed the following parameters: $\sigma=1.79 \times 10^{3} \mathrm{~W} / \mathrm{m}^{2} K^{4}, S=$ $2.89 \times 10^{13} \mathrm{~W} / \mathrm{m}^{2}$ and $c=3.0 \times 10^{13} \mathrm{~J} / \mathrm{m}^{2} \mathrm{~K}\left(10^{3} \mathrm{y}\right)$. Note that time scale was changed from seconds to thousands of years. Results of the daisyworld with climate variability are compared to those obtained without this variability, with just linear increase of the luminosity $\left(L_{0}=\omega=0\right)$. Figure 12 presents the temperature evolution of the daisyworld showing a comparison between the situation without (represented by light line) and with (represented by dark line) climate variability. The effect of the greenhouse gases is also of concern. The left panel presents results without greenhouse gases while the right panel considers a situation with greenhouse gases. Both systems present the same general tendency but the daisyworld with climate variability presents results with irregular behavior.
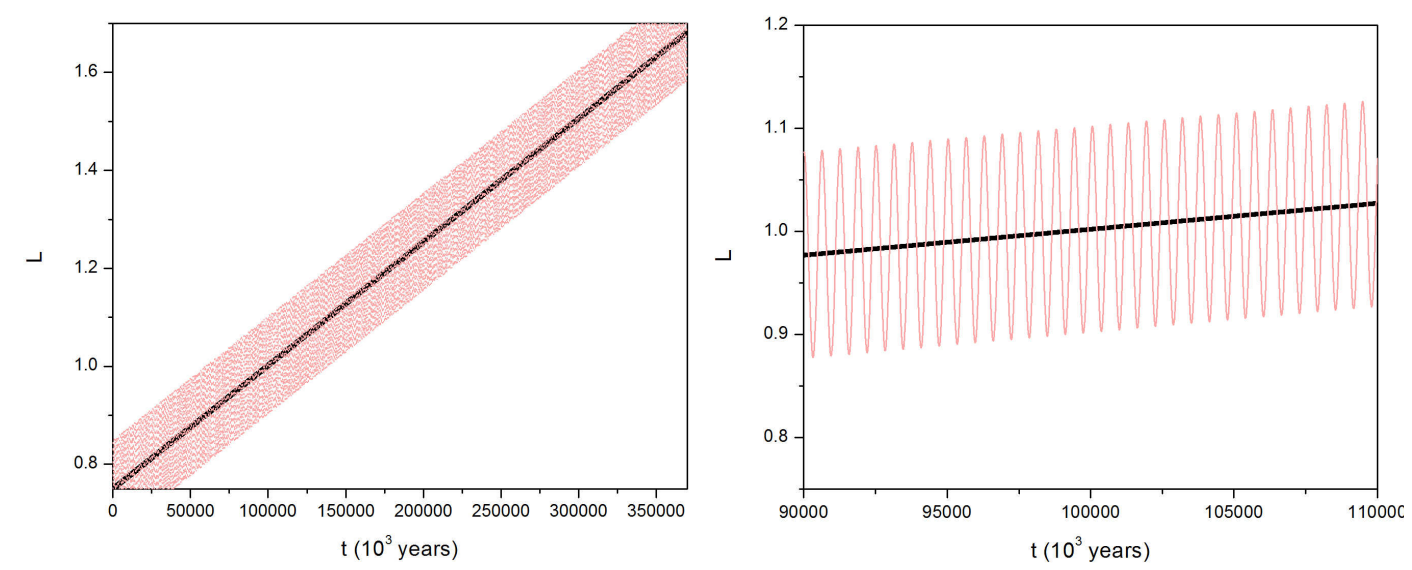

Figure 11. Linear luminosity (dark line) and sinusoidal variation (light line) 


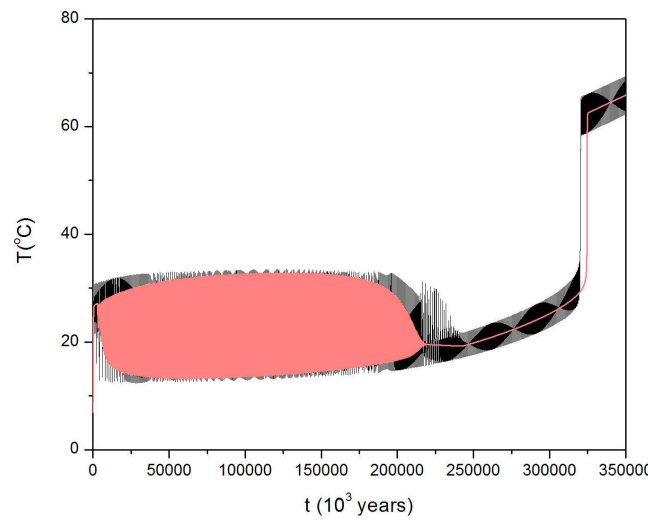

(a)

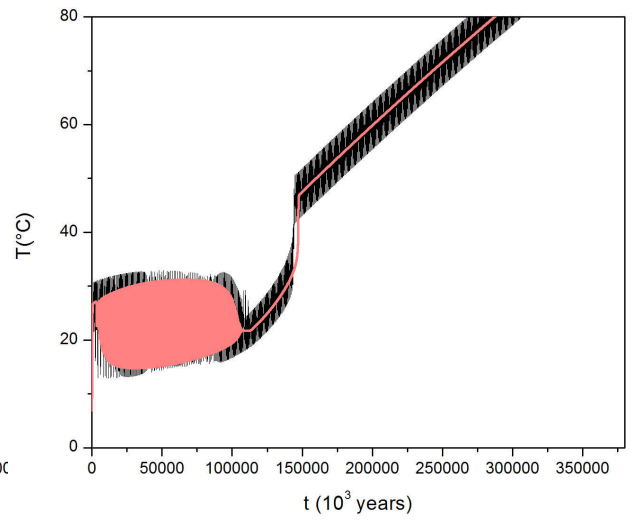

(b)

Figure 12. Temperature evolution due to solar luminosity linear increase. $L_{0}=0.1$ and $\omega=0.01$ (dark line), $L_{0}=0.0$ and $\omega=0.0$ (light line) without $(a)$ and with $(b)$ greenhouse gases variable

$$
(0.0 \leq G \leq 0.8)
$$

Figure 13 presents the correspondent evolution of the daisy populations for the same cases. Once again, it should be observed that the system has an irregular behavior when climate variability is considered. Chaotic behavior of the daisyworld was addressed in different references being a controversial subject (Wood, 2008).

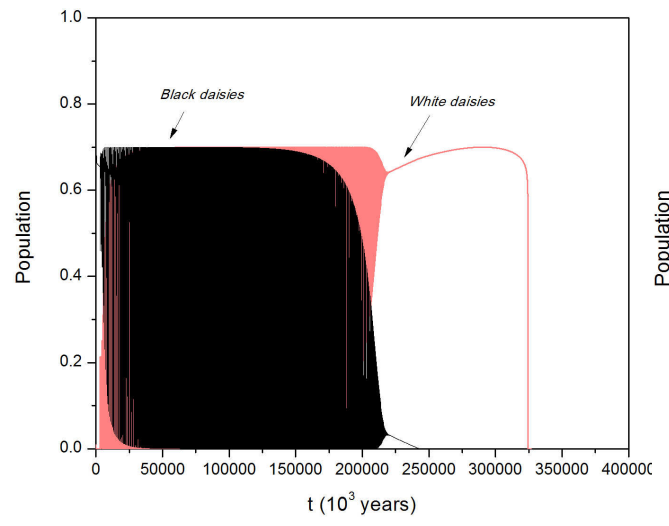

(a)

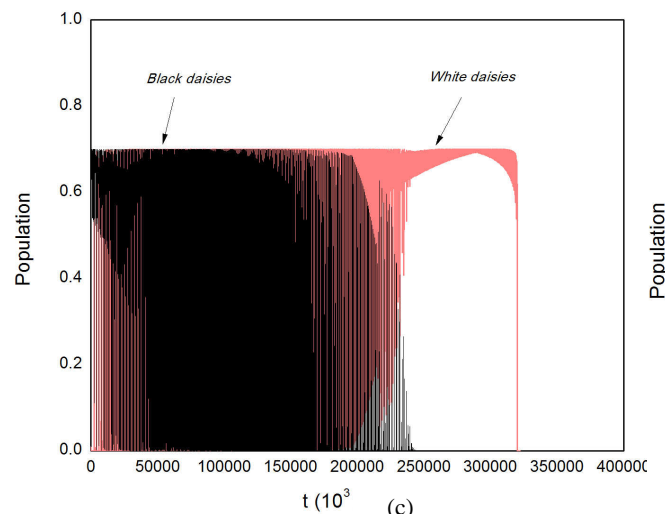

(c)

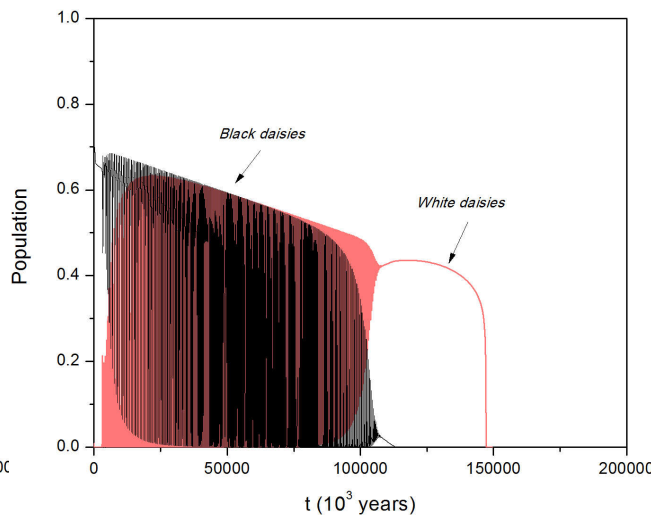

(b)

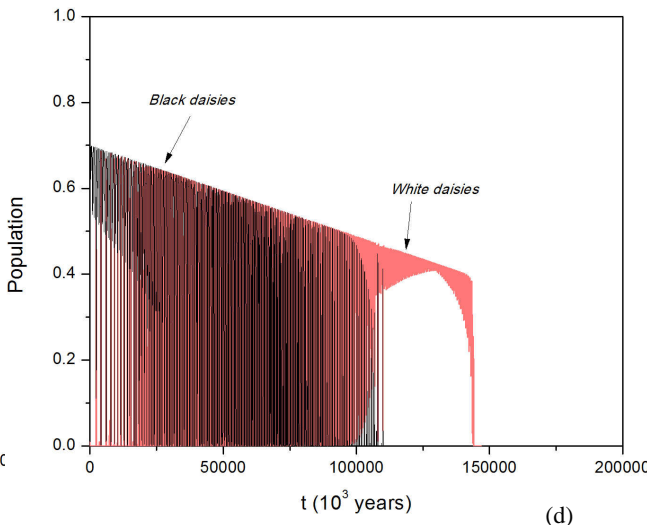

(d)

Figure 13. Figure 13. Daisy populations evolution with solar luminosity linear increase. (a) $L_{0}=0.0, \omega=0.0$ and $G=0$; (b) $L_{0}=0.0, \omega=0.0$ and $(0.0 \leq G \leq 0.8)$; (c) $L_{0}=0.1$ and $\omega=0.01, G=0$; (d) $L_{0}=0.1, \omega=0.01$ and $(0.0 \leq G \leq 0.8)$ 
The irregular behavior presented in the previous simulations motivates a deeper dynamical investigation of the daisyworld. Therefore, it is considered the influence of some parameters in the system dynamics. The analysis starts with the bifurcation diagram that presents stroboscopically sampled temperature values under the slow quasi-static increase of the constant luminosity. This diagram demonstrates the influence of the parameter on system dynamics, showing its global behavior. Initially, it is assumed that $G=0.21052, L_{0}=0.1, \omega=0.01$ and constant luminosity $\bar{L}$ is varying from 0.75 to 1.2 . Figure 14 presents this bifurcation diagram that shows regions related to single points as well as regions associated with cloud of points. In order to evaluate the influence of other parameters, different bifurcation diagrams are plotted, for different, constant parameters.

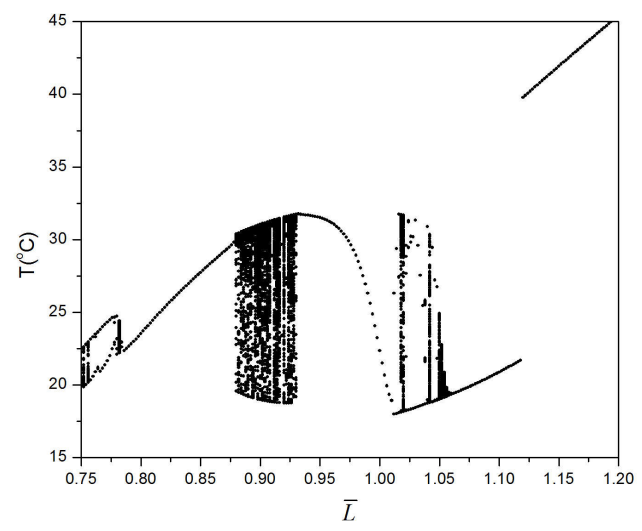

Figure 14. Bifurcation diagram varying $\bar{L}(0.75 \leq \bar{L} \leq 1.20)$ with $\omega=0.01$

The influence of frequency parameter $\omega$ may be evaluated by considering bifurcation diagrams presented in Figure 15 for different, constant values of $\omega: \omega=0.05$ and $\omega=$ 0.1 . Note that this change can dramatically alter the general system dynamics.

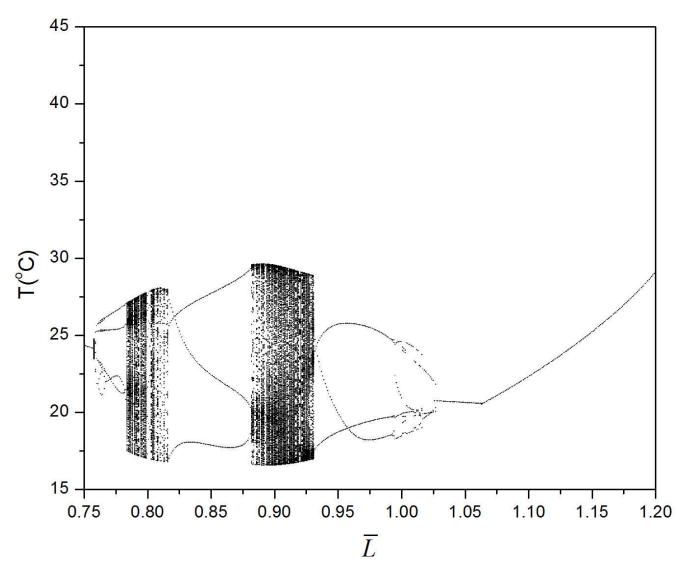

(a)

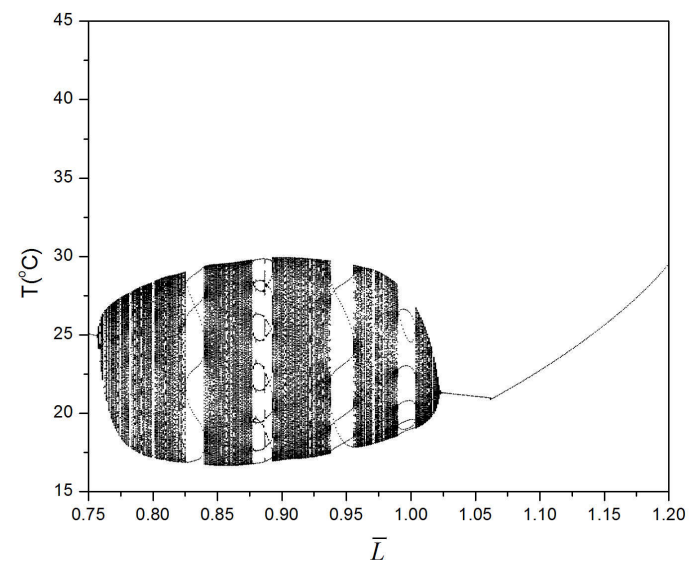

(b)

Figure 15. Bifurcation diagram varying $\bar{L}:(a) \omega=0.05$ and $(b) \omega=0.1$ 
The influence of climate variability parameter is evaluated by considering different values of the amplitude of the sinusoidal luminosity, $L_{0}: L_{0}=0.1$ and $L_{0}=2.0$. Figure 16 presents bifurcation diagrams for these situations and, once again, it is clear that the change of this parameter can dramatically alter the system dynamics. Greenhouse gases also influence system dynamics and situation with different values of $G(G=0.31$ and $G=0.46$ ) are presented in Figure 17. Under these conditions, the system tends to be more regular, accelerating the end of the balance between life and environment.

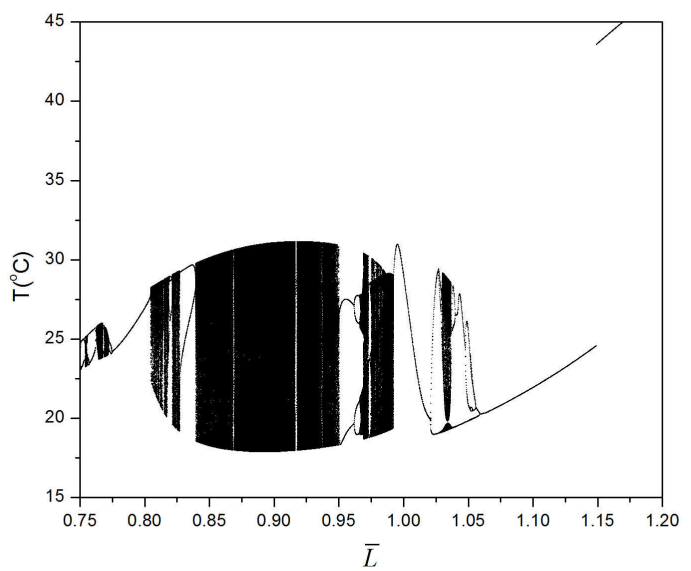

(a)

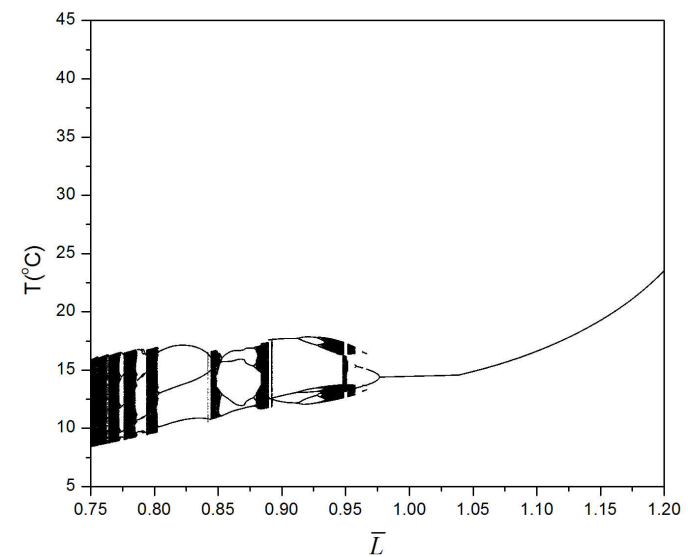

(b)

Figure 16. Bifurcation diagram varying $\bar{L}:$ (a) $L_{0}=0.05$ and (b) $L_{0}=2.0$

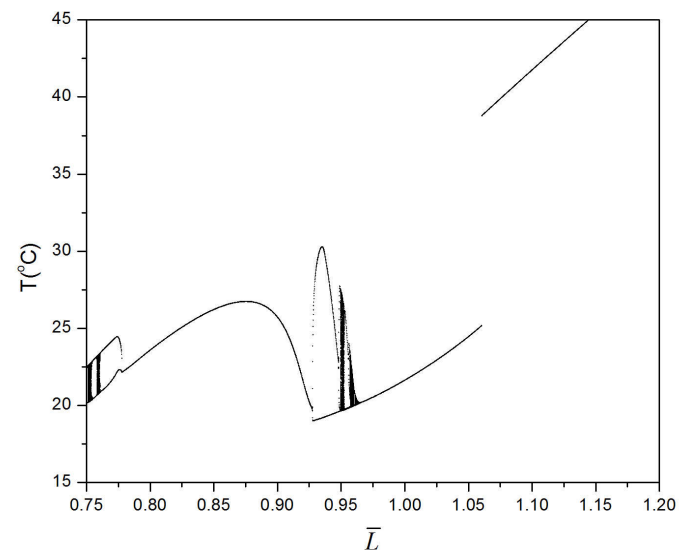

(a)

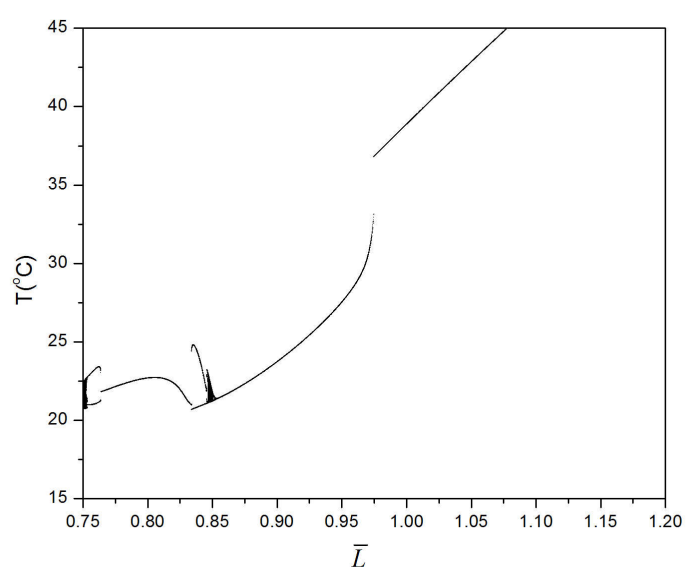

(b)

Figure 17. Bifurcation diagram varying $\bar{L}:$ (a) $G=0.31$ and (b) $G=0.46$

The details of the system dynamics are now in focus by revisiting results presented in Figure 14. Figure 18 presents enlargements of the bifurcation diagram for different ranges of constant luminosity. Note that bifurcation and chaos occur in the daisyworld dynamics. 

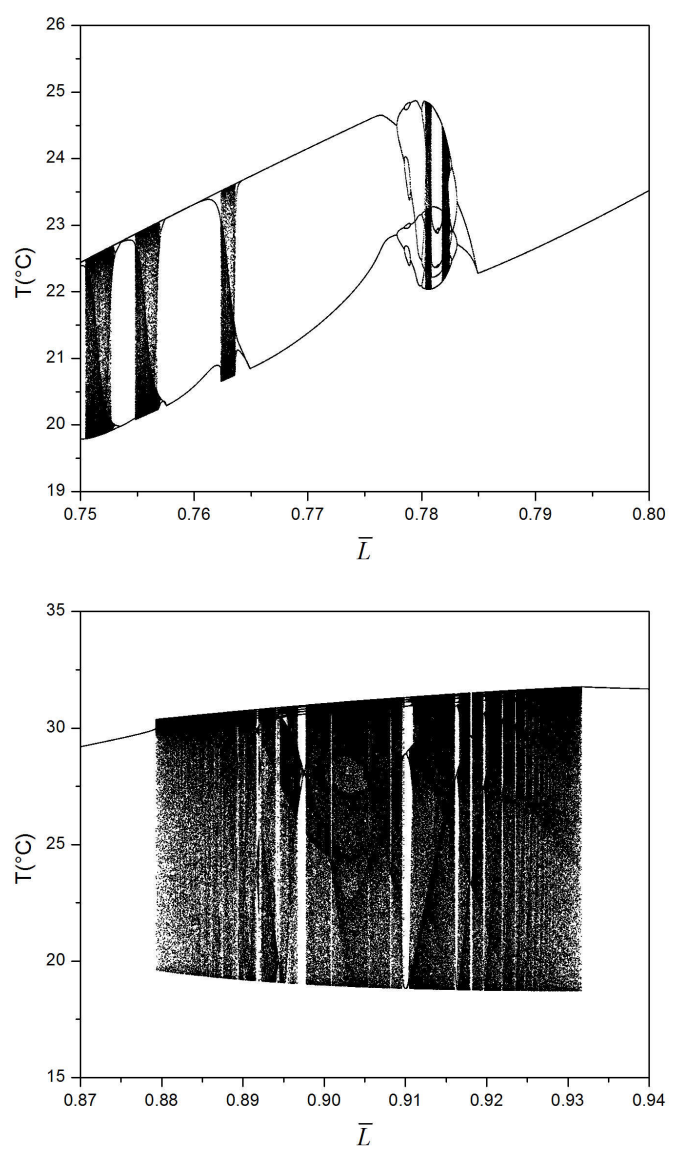

(b)

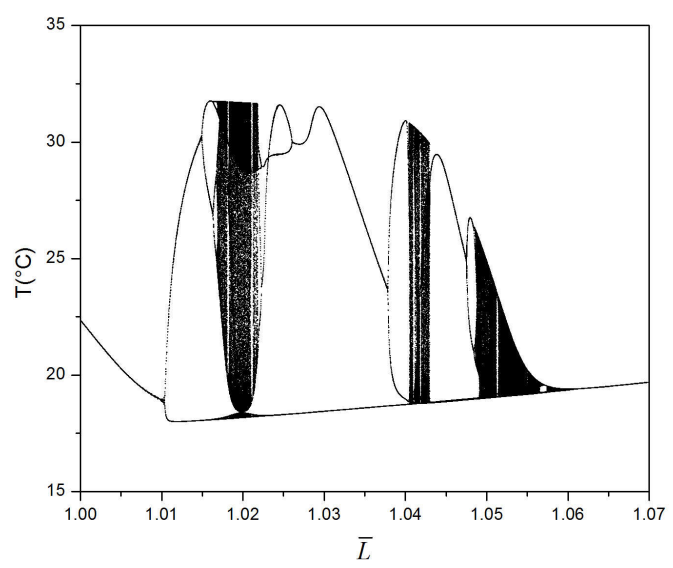

(c)

Figure 18. Bifurcation diagram varying $\bar{L}$. (a) $0.75 \leq \bar{L} \leq 0.80$; (b) $0.87 \leq \bar{L} \leq 0.94$ and (c)

$$
1.00 \leq \bar{L} \leq 1.07
$$

Based on these bifurcation diagrams, a detailed investigation of the daisyworld behavior is performed analyzing system responses for different sets of parameters, changing the value of $\bar{L}$. Figures 19-33 show some of these behaviors presenting the state space and Poincaré section (left panel) and temperature time series (right panel). Let us start by considering a period-2 response obtained when $\bar{L}=0.76$. Figure 19 shows this periodic response represented by a closed curve in the phase space and by two points in the Poincaré section. The temperature time series is represented by a 
regular pattern that repeats for each period. This behavior is followed by a chaotic-like behavior when $\bar{L}=0.7629$ (Figure 20). This response has a complex behavior in the phase space and a cloud of points in the Poincaré section. Moreover, it should be observed an irregular pattern for temperature time series.
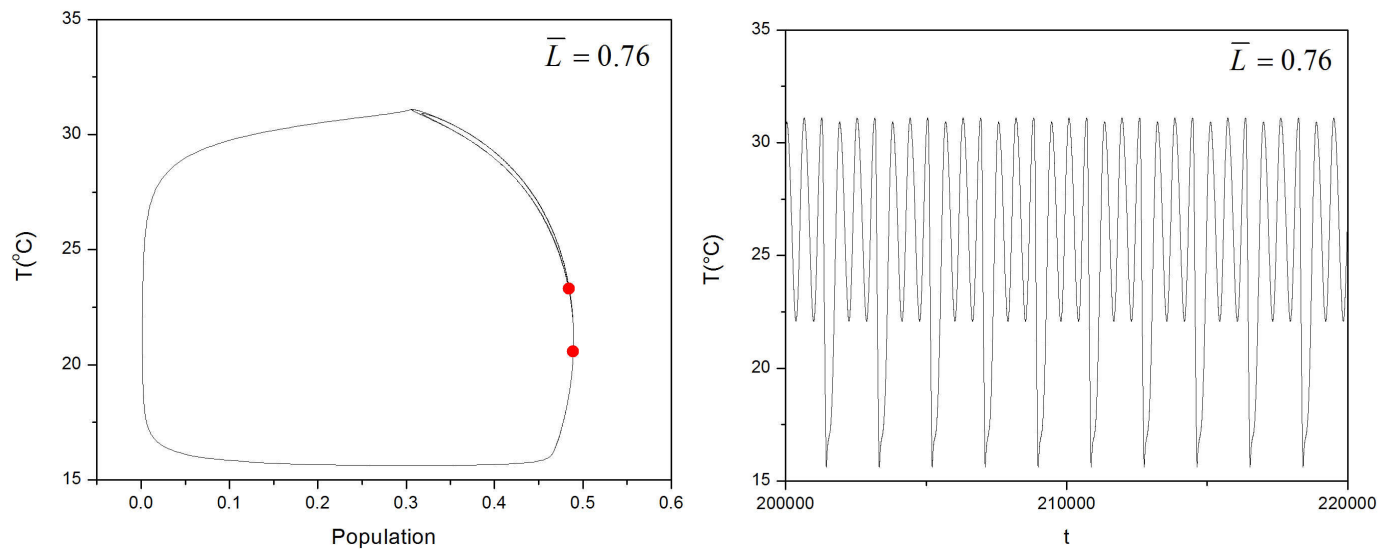

Figure 19. Period-2 response for $\bar{L}=0.76$. State space and Poincaré section (left); temperature evolution (right)
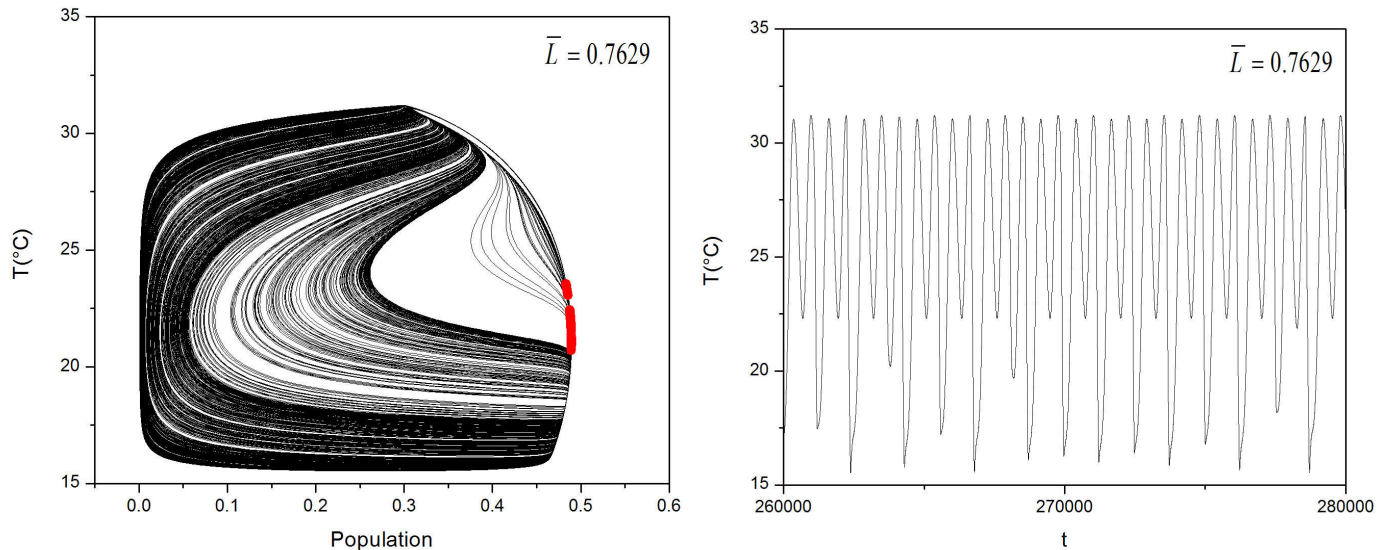

Figure 20. Chaotic behavior for $\bar{L}=0.7629$. State space and Poincaré section (left); temperature evolution (right)

By increasing the luminosity to $\bar{L}=0.7787$ (Figure 21 ), the system presents a period- 8 response that is followed by a period- 4 when $\bar{L}=0.78$ (Figure 22). Both situations have the same qualitative characteristics presenting a closed curve in phase space and a finite number of points in the Poincare section. The increase of the luminosity to $\bar{L}=0.782$ induces a new chaotic-like response, different from the previous one (Figure 23). 

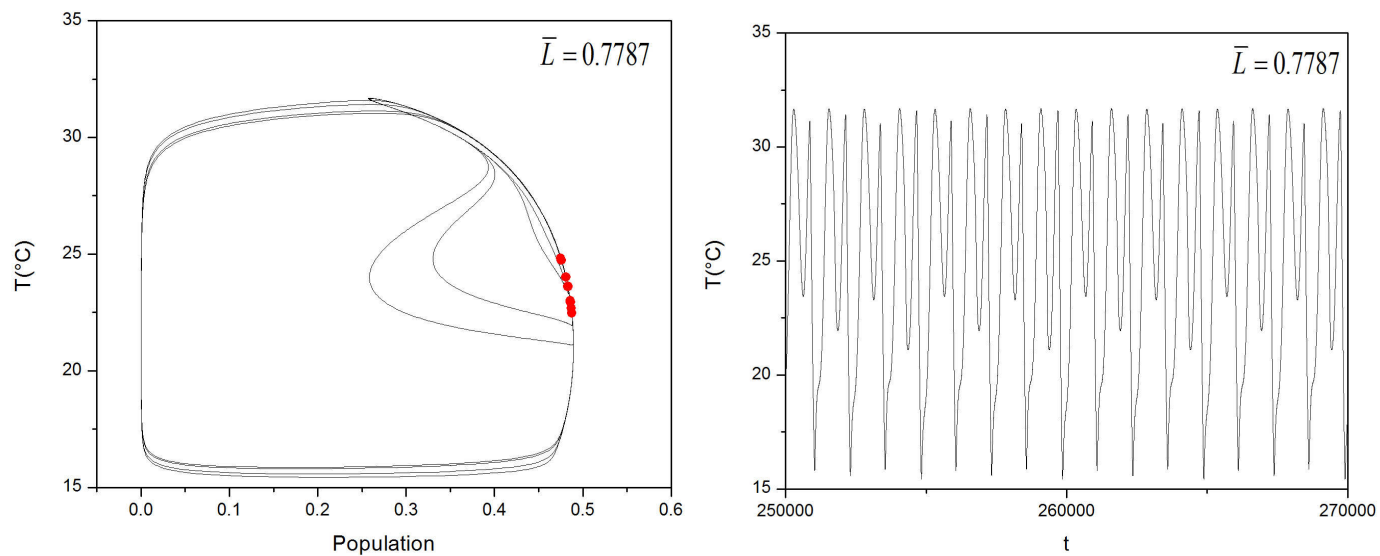

Figure 21. Period-8 behavior for $\bar{L}=0.7787$. State space and Poincaré section (left); temperature evolution (right)
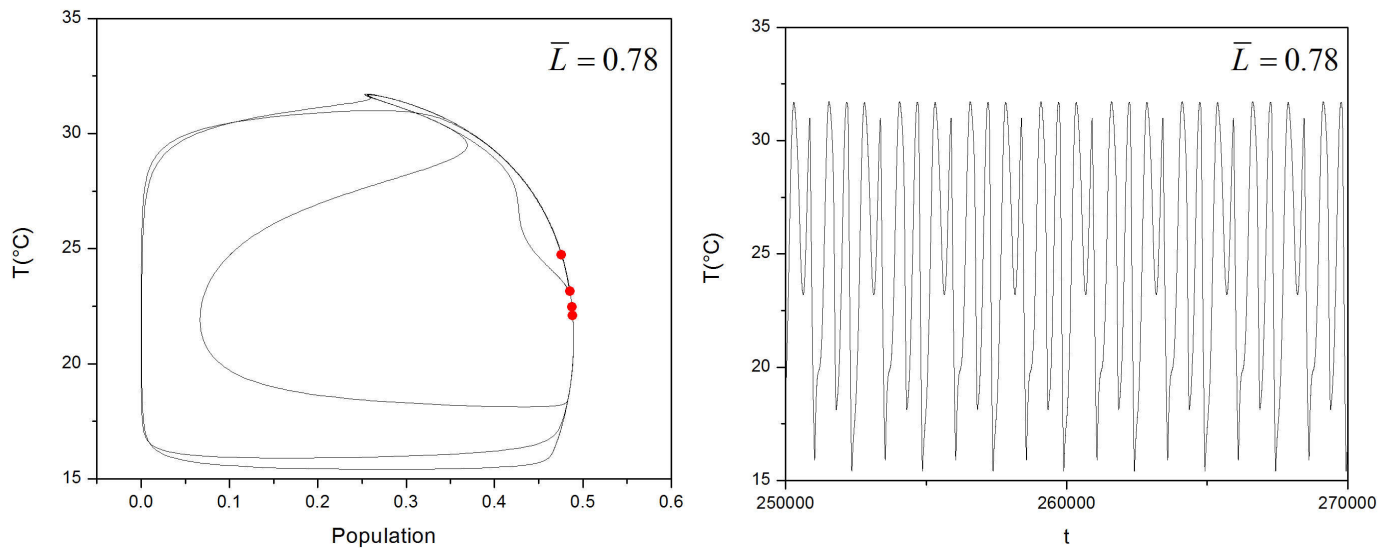

Figure 22. Period-4 behavior for $\bar{L}=0.78$. State space and Poincaré section (left); temperature evolution (right)
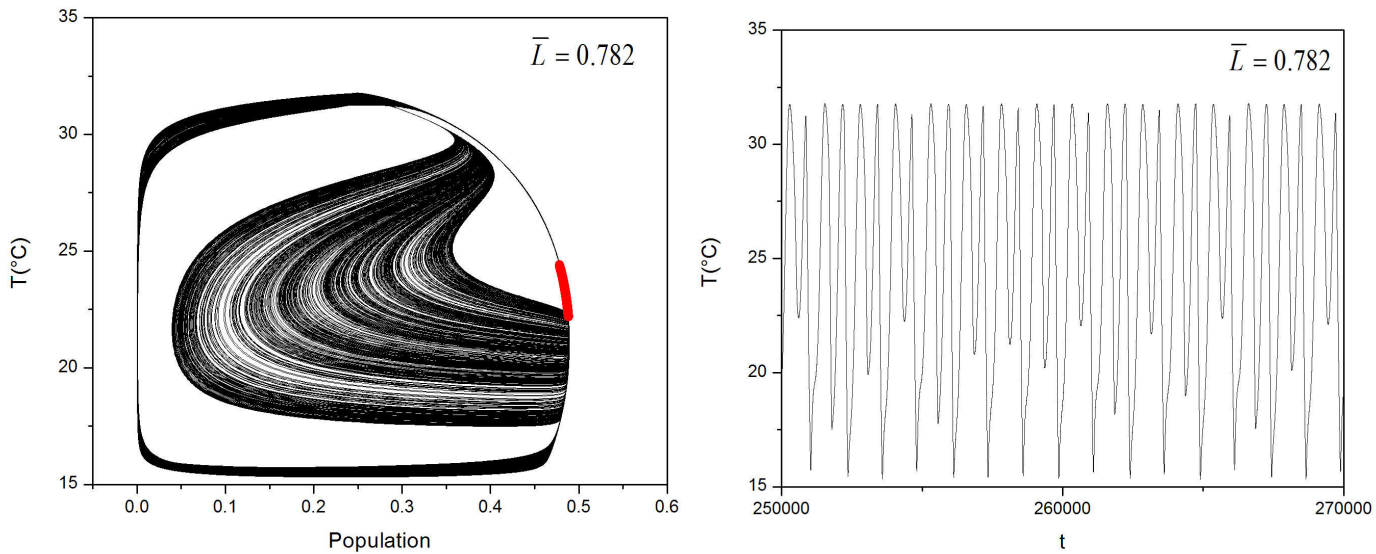

Figure 23. Chaotic behavior for $\bar{L}=0.782$. State space and Poincaré section (left); temperature evolution (right) 
A sequence of bifurcations occurs until $\bar{L}=0.785$ when a period-1 response appears. In the range between 0.88 and 0.93 , quasi-periodic behavior emerges as when $\bar{L}=0.90$ (Figure 24). Periodic windows are also present in this range as can be observed for the period-3, $\bar{L}=0.9082$ (Figure 25) and period-7, $\bar{L}=0.9097$ (Figure 26).
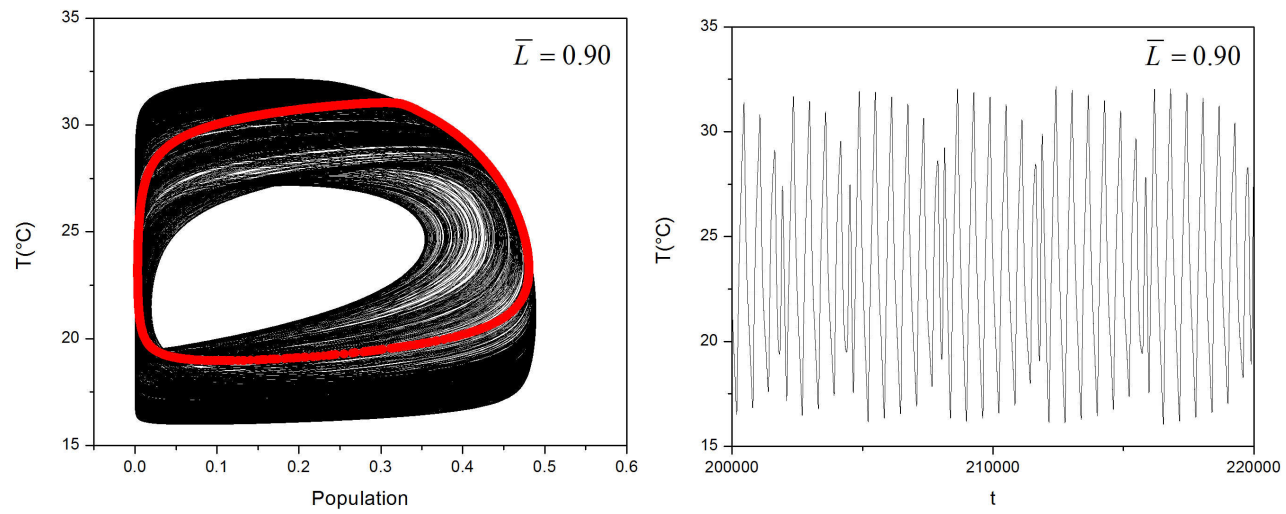

Figure 24. Quasi-periodic behavior for $\bar{L}=0.90$. State space and Poincaré section (left); temperature evolution (right)
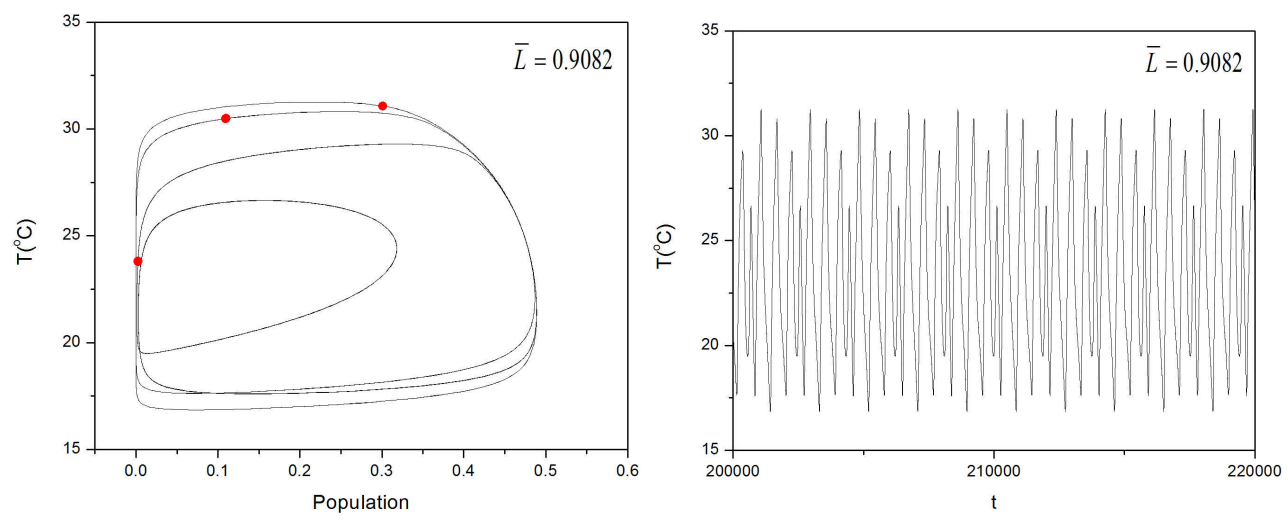

Figure 25. Period-3 behavior for $\bar{L}=0.9082$. State space and Poincaré section (left); temperature evolution (right)
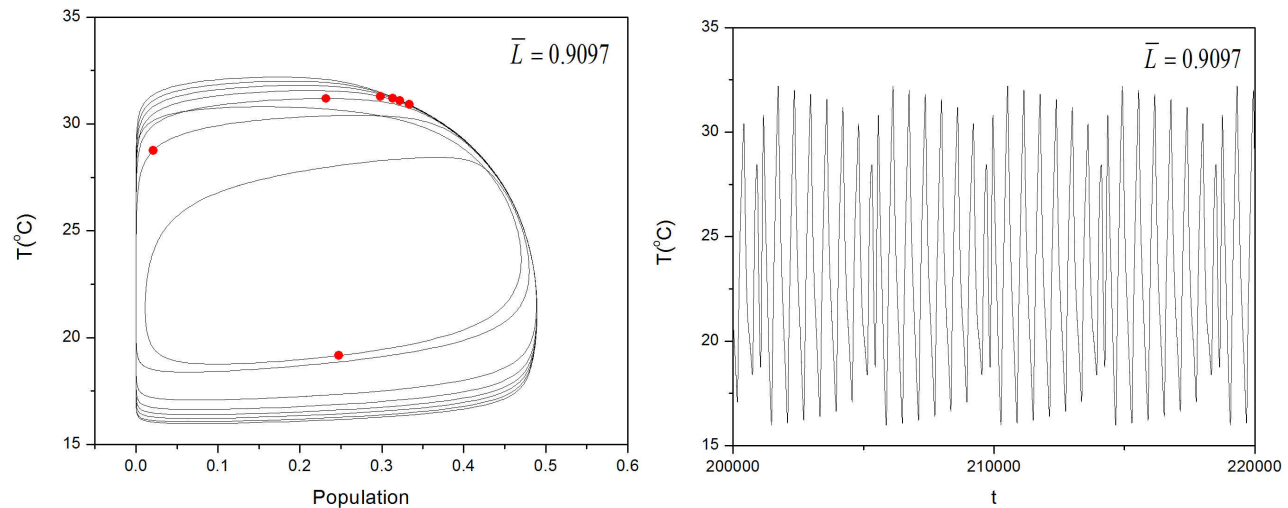

Figure 26. Period-7 behavior for $\bar{L}=0.9097$. State space and Poincaré section (left); temperature evolution (right) 
Afterwards, chaotic-like behavior occurs again as can be observed for $\bar{L}=0.919$ (Figure 27) and $\bar{L}=0.9238$ (Figure 28). Once again, it is important to observe the structure of the Poincaré section and also the irregular behavior related to time series.
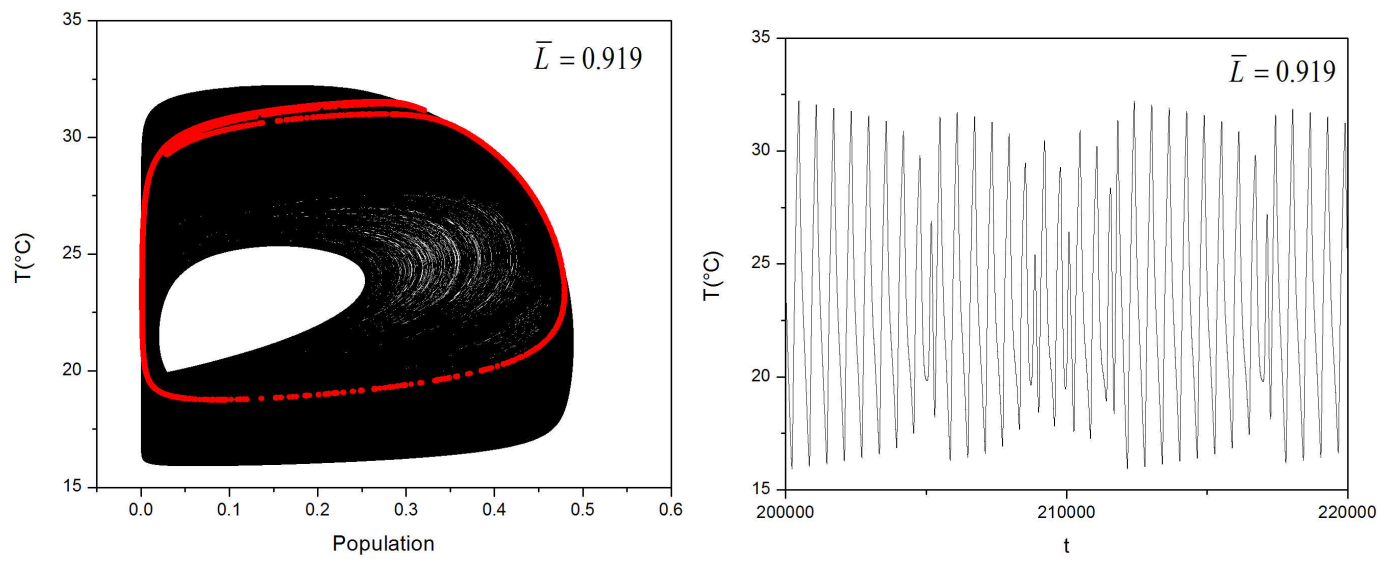

Figure 27. Chaotic behavior for $\bar{L}=0.919$. State space and Poincaré section (left); temperature evolution (right)
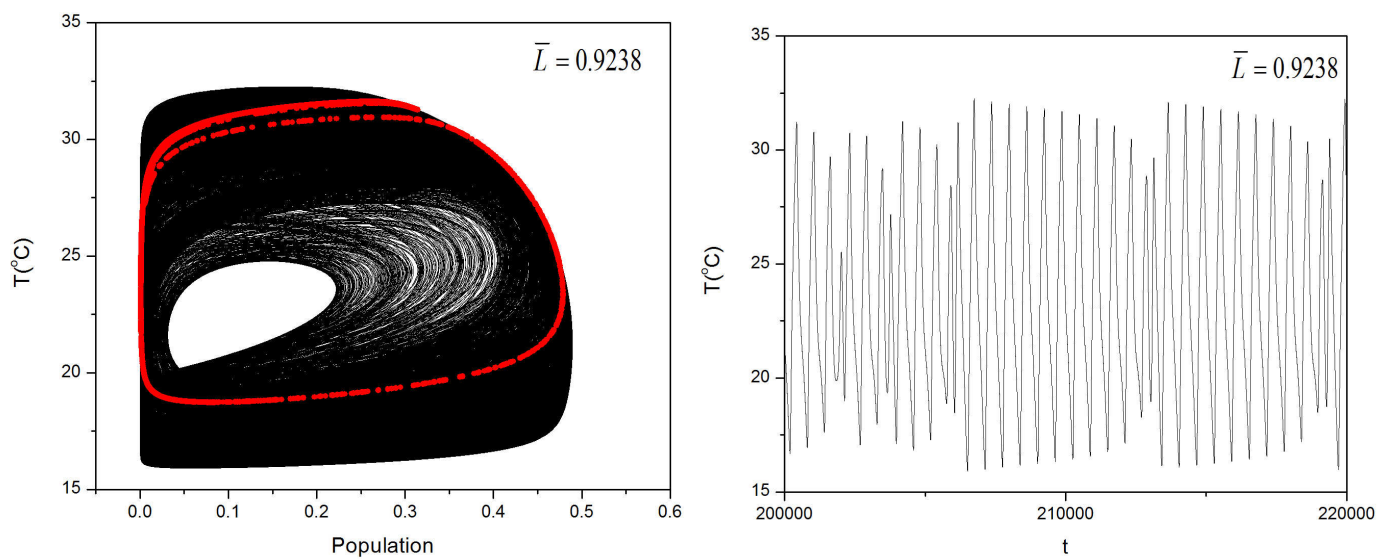

Figure 28. Chaotic behavior for $\bar{L}=0.9238$. State space and Poincaré section (left); temperature evolution (right)

In the range between 0.9328 and 1.01 the system presents new bifurcations. For $\bar{L}=$ 1.01, a period-1 response occurs (Figure 29). Some bifurcations make the system increases periodicity reaching a chaotic regime as for $\bar{L}=1.02$ (Figure 30 ). For $\bar{L}=$ 1.04, a period-3 response occurs (Figure 31) and a chaotic-like response occurs again for $\bar{L}=1.05$ (Figure 32). For values greater than 1.06 the system presents period-1 response. 

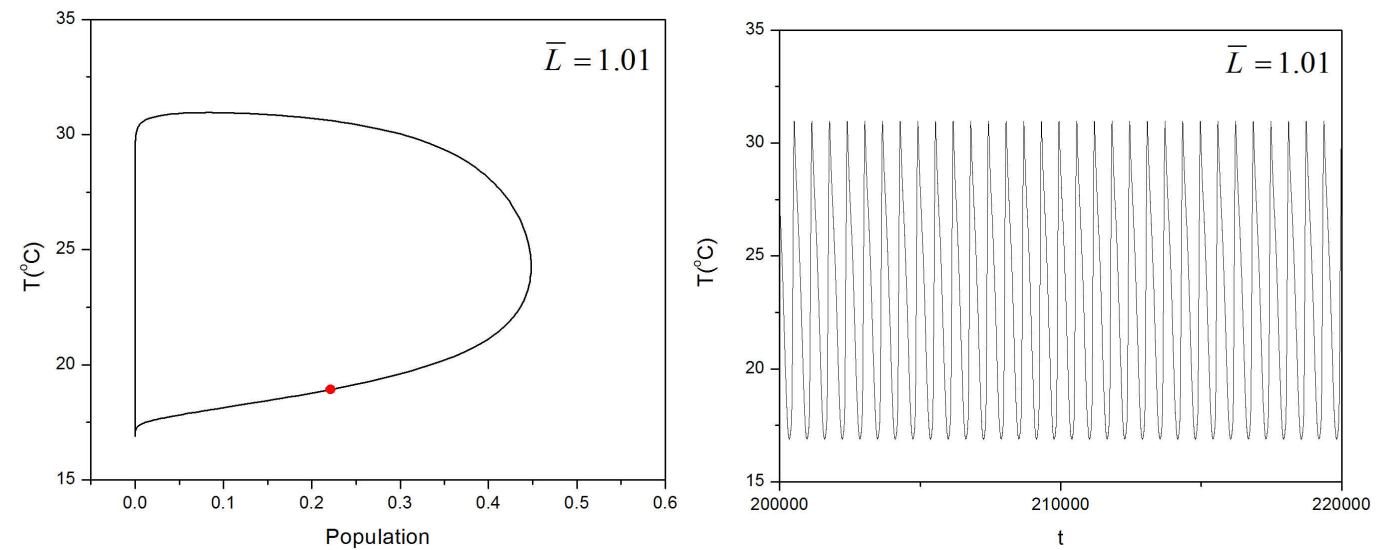

Figure 29. Period-1 behavior for $\bar{L}=1$.01. State space and Poincaré section (left); temperature evolution (right)
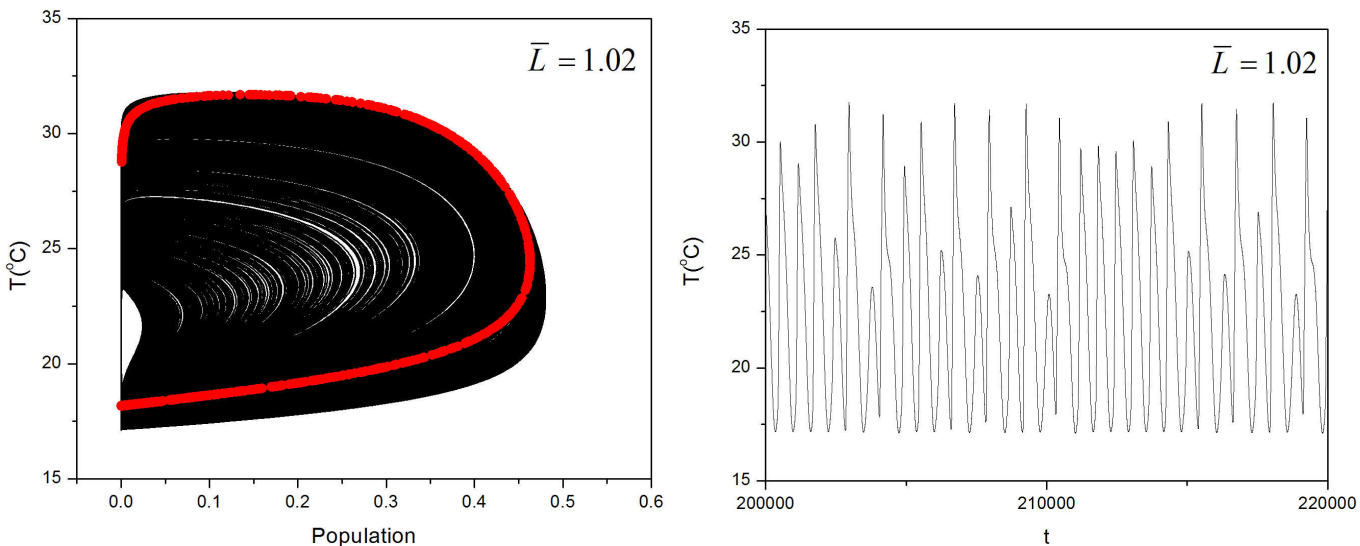

Figure 30. Chaotic behavior for $\bar{L}=1.02$. State space and Poincaré section (left); temperature evolution (right)
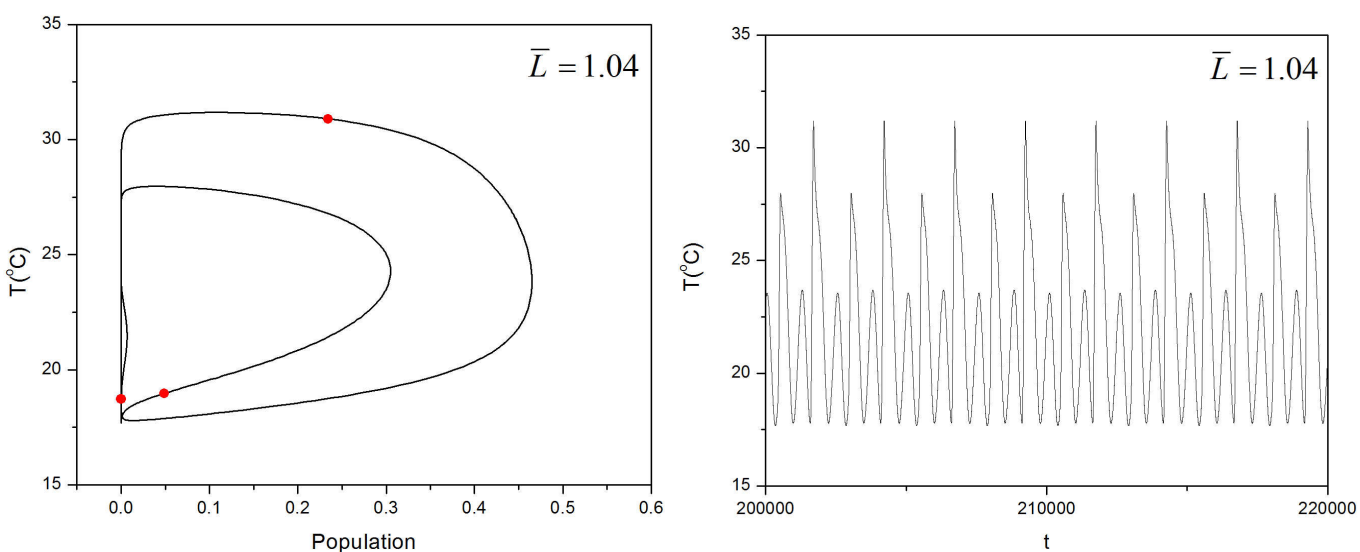

Figure 31. Period-3 behavior for $\bar{L}=1.04$. State space and Poincaré section (left); temperature evolution (right) 

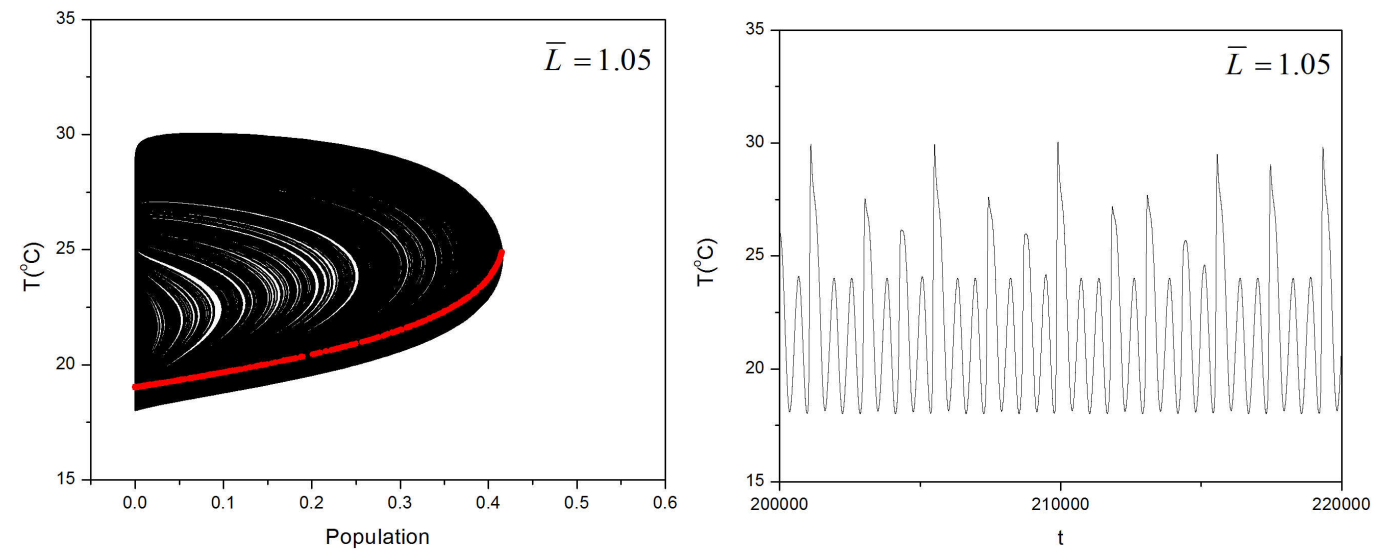

Figure 32. Chaotic behavior for $\bar{L}=1.05$. State space and Poincaré section (left); temperature evolution (right)

At this point, it is clear the rich dynamics related to the daisyworld. Bifurcations and chaos are present and the existence of chaotic behavior is of special interest due to instability issues. The comprovation of this kind of behavior requires the use of some diagnostic tool. Lyapunov exponents represent one of the most acceptable diagnostic tool of chaos. They measure the local divergence of nearby orbits and a system containing at least one positive exponent is chaotic. There are many possibilities to evaluate Lyapunov exponents (Franca \& Savi, 2003, 2001). Here, the algorithm due to Kantz (1994) is employed to temperature time series related to Poincaré map. This algorithm establishes that the divergence rate trajectories fluctuates along the trajectory, with the fluctuation given by the spectrum of effective Lyapunov exponents. The determination of the maximum Lyapunov exponent due to Kantz algorithm is related to the slope of the curve $S(\delta)$ that represents distances as a function of a relative time associated with local divergence.

Figures 33-35 presents Poincaré sections and the associated maximum Lyapunov exponent for some daisyworld responses. Basically, three different situations are of concern: period-8 response presented in Figure $21(\bar{L}=0.7787)$; chaotic response presented in Figure $28(\bar{L}=0.9238)$; and other chaotic response presented in Figure 32 ( $\bar{L}=1.05)$. Figure 33 shows a period- 8 response for $\bar{L}=0.7787$. It should be pointed out that the Poincaré section is related to 8 points and the Lyapunov exponent curve presents a zero-slope curve meaning that the maximum exponent is null $(\lambda=0)$. 

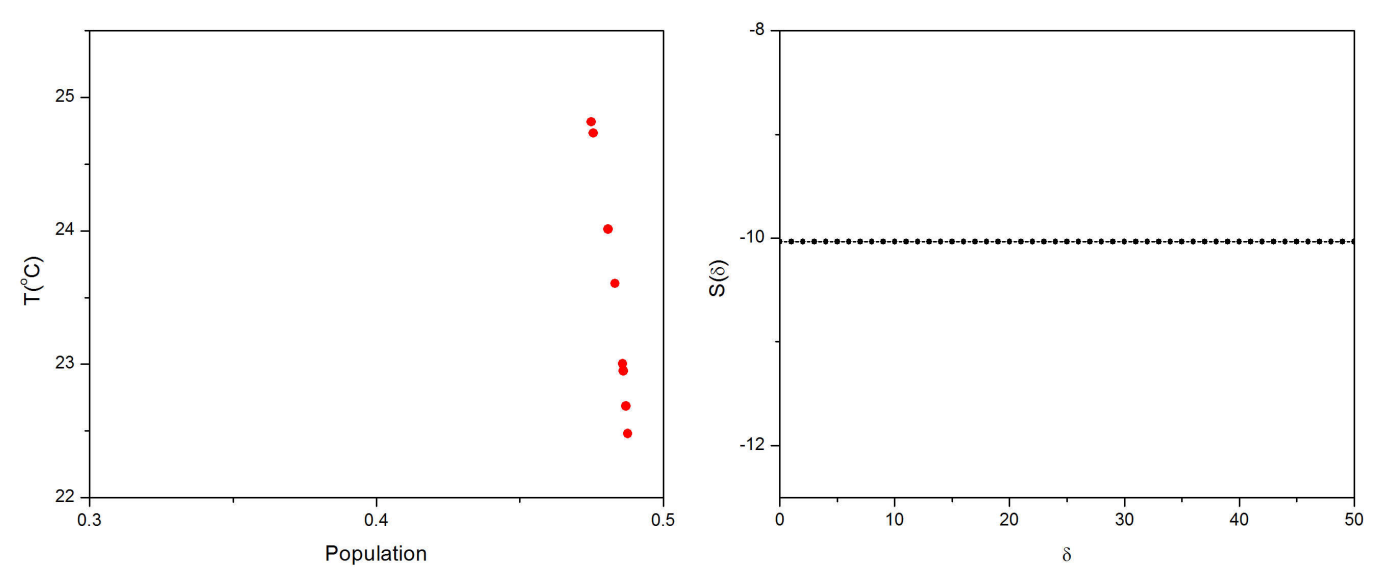

Figure 33. Period-8 behavior for $\bar{L}=0.7787$ (same situation presented in Figure 24). Poincaré section (left) and respective maximum Lyapunov exponents (right)

Chaotic response is now in focus. Figure 34 considers the situation where solar luminosity is $(\bar{L}=0.9238)$. Under this condition, the Poincaré section presents a fractal-like structure and the maximum Lyapunov exponent is related to a positive slope $(\lambda=0.28)$. A different chaotic behavior is presented in Figure 35 associated with a situation where $\bar{L}=1.05$. Once again, a fractal-like structure is observed in the Poincaré section and a positive slope confirms a positive maximum Lyapunov exponent $(\lambda=0.36)$.
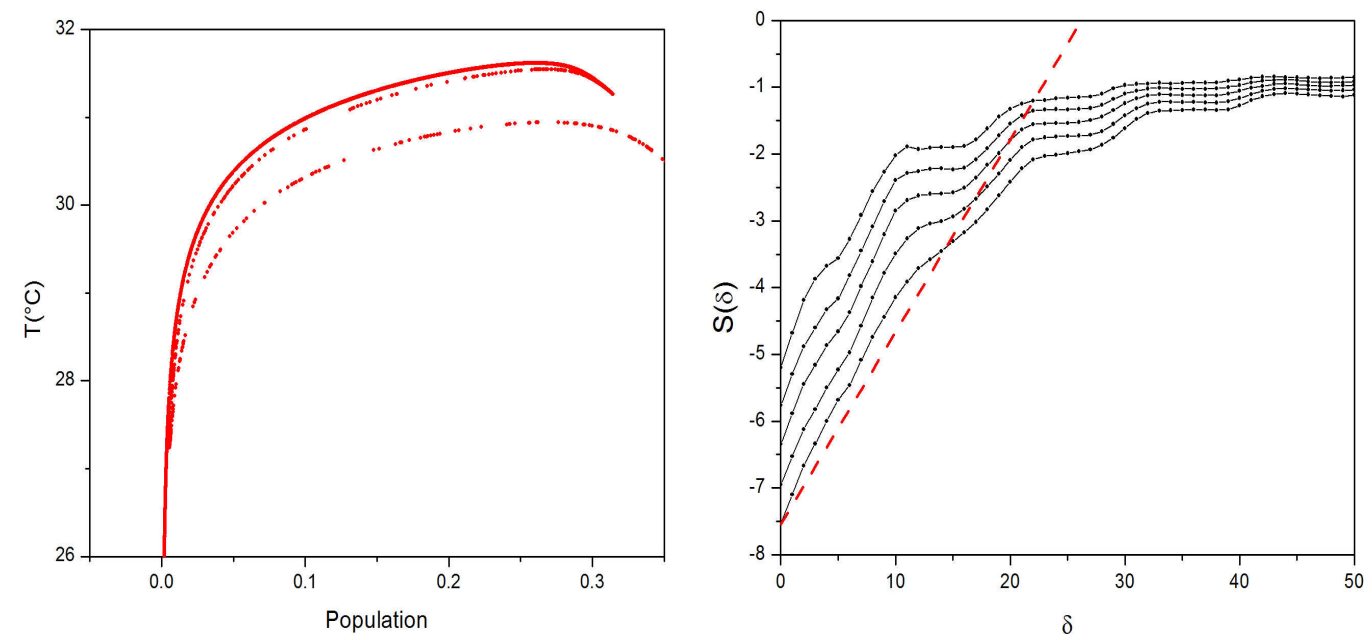

Figure 34. Chaotic behavior for $\bar{L}=0.9238$ (same situation presented in Figure 32). Poincaré section (left) and respective maximum Lyapunov exponents (right) 

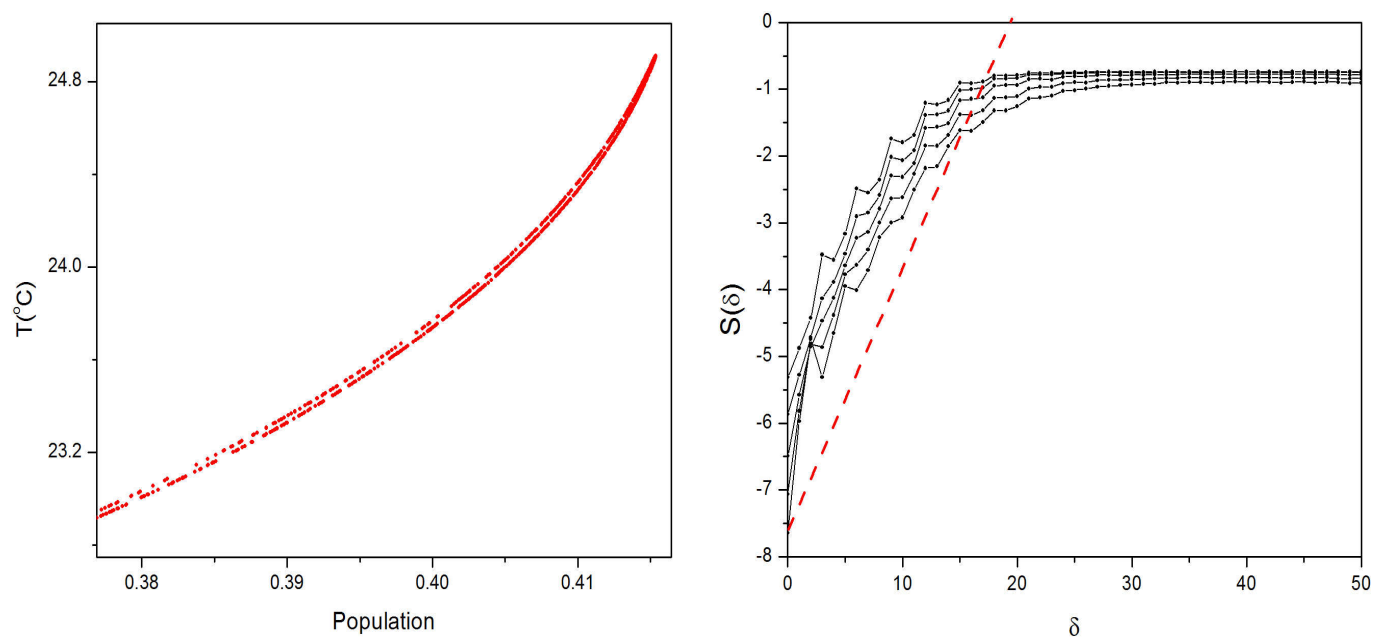

Figure 35. Chaotic response for $\bar{L}=1.05$ (same situation presented in Figure 36). Poincaré section (left) and respective maximum Lyapunov exponent (right)

Chaotic behavior has an intrinsic order and one of its most remarkable characteristics is the sensitivity dependence to initial conditions. In this regard, we revisit the three distinct situations previous analyzed to observe this behavior: period-8 response presented in Figure 21 and $33(\bar{L}=0.7787)$; chaotic response presented in Figure 28 and $34(\bar{L}=0.9238)$; and other chaotic response presented in Figure 32 and 35 ( $\bar{L}=$ 1.05). The analysis considers a variation of $1 \%$ in initial conditions assuming two different temperatures: $280 \mathrm{~K}$ and $282.8 \mathrm{~K}$. The idea is to compare both responses evaluating the system sensitivity. Figure 36-38 presents this comparison monitoring the temperature and the daisy populations. Figure 36 presents this comparison for the period-8 response $(\bar{L}=0.7787)$. As expected for periodic responses, this perturbation does not have significant influence on system response and therefore, temperature and daisy populations are essentially the same for both cases. By analyzing chaotic behavior, the influence of this perturbation is quite different. Figure 37 presents the system response for $\bar{L}=0.9238$ while Figure 38 presents response for $\bar{L}=1.05$. In both cases, it is possible to observe a significant different response for distinct initial conditions. Nevertheless, it is also important to highlight the stability aspect of the daisyworld. Although the system has sensitive dependence to initial conditions and eventually, presents strong divergences due to the initial perturbation, there is a general tendency to the stability in terms of global behavior. Our argue is based on the observation that the system response is basically the same during most of the time, eventually changing dramatically, but then returning to the original behavior. Another aspect that should be pointed out is that the system response for $\bar{L}=1.05$ (Figure 38) presents more dramatic changes when compared to those for $\bar{L}=0.9238$ (Figure 37). This is also expected since the maximum Lyapunov exponent for the first case is greater than the second one and therefore, has greater divergence of nearby orbits. 

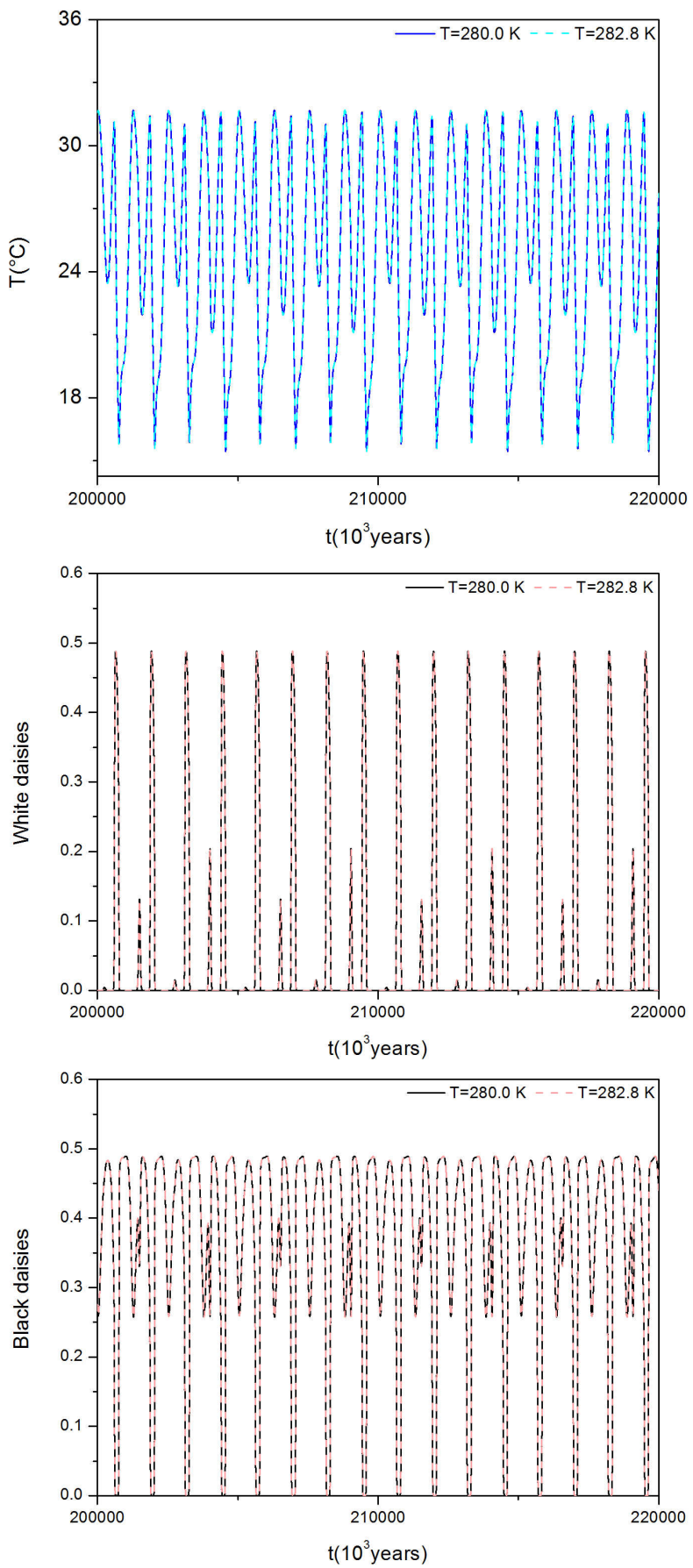

Figure 36. Sensitivity to initial conditions: period-8 response for $\bar{L}=0.7787$ (same situation presented in Figures 21 and 33) 

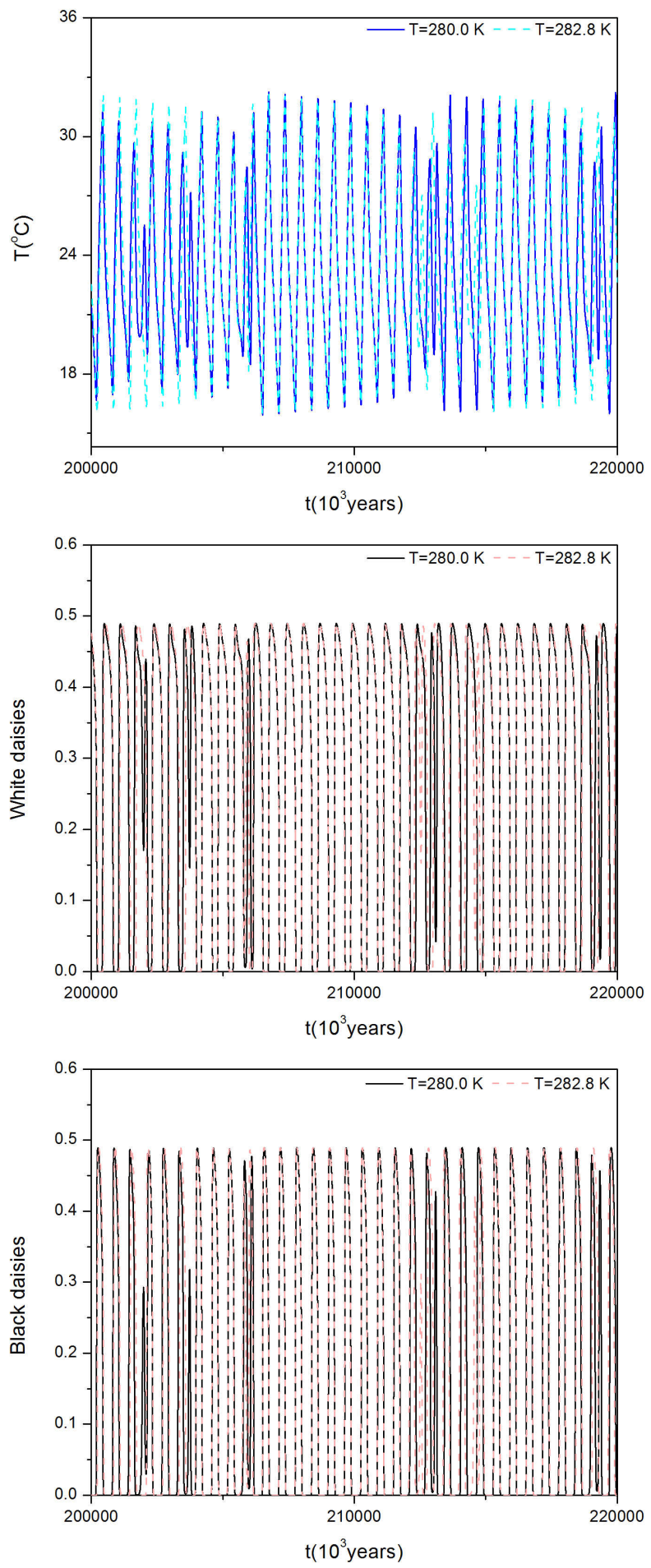

Figure 37. Sensitivity to initial conditions: chaotic response for $\bar{L}=0.9238$ (same situation presented in Figures 28 and 34) 

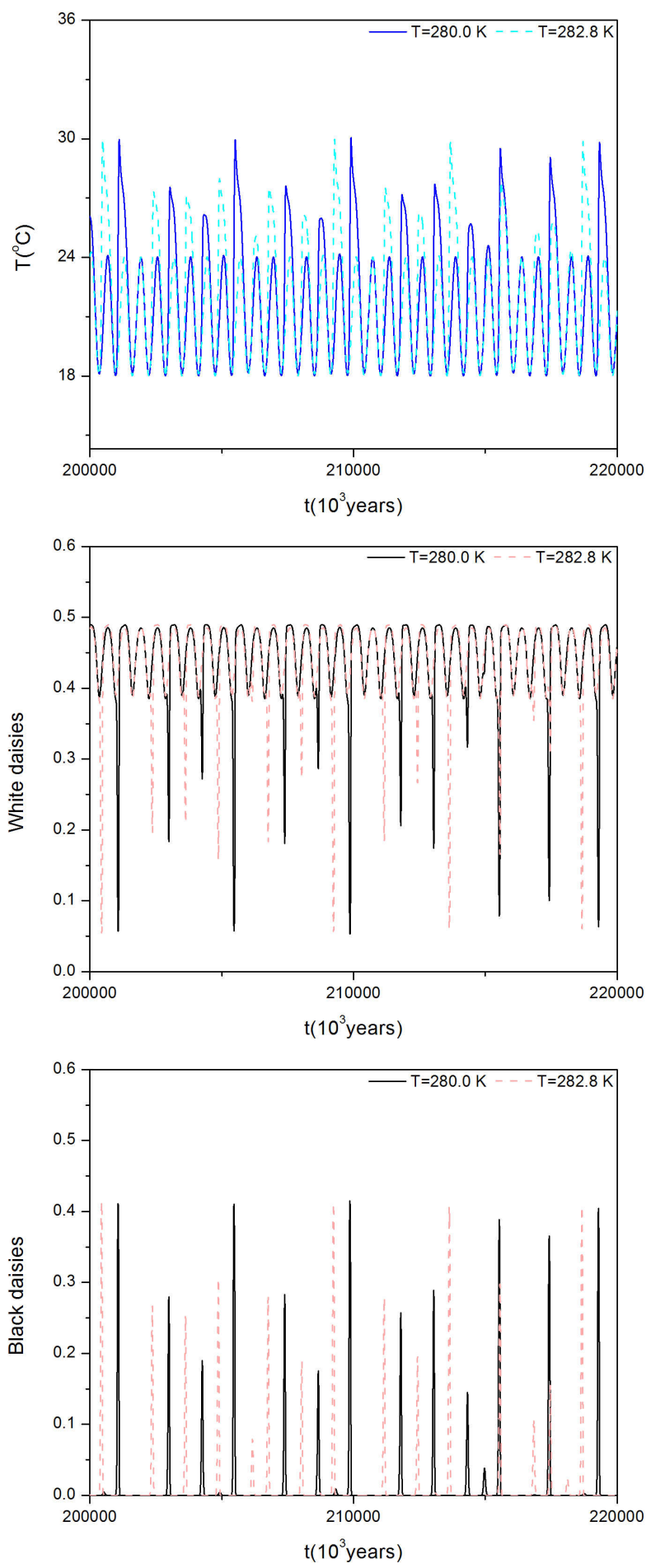

Figure 38. Sensitivity to initial conditions: chaotic response for $\bar{L}=1.05$ (same situation presented in Figures 32 and 35)

\section{Conclusions}

Daisyworld is an archetypal model of the Earth being able to describe the global regulation that can emerge from the interaction between life and environment. In brief, daisyworld represents life by black and white daisy populations while the environment 
is represented by temperature. An extra variable related to greenhouse gases is incorporated into the model allowing the analysis of the global warming. This variable is prescribed as a time series, being known by some measurement. Besides, energy equation is considered in order to investigate transient phenomena related to temperature variation. The climate variability is described by considering a sinusoidal variation of the solar luminosity. A general analysis of the daisyworld is carried out analyzing constant and linear increase of the solar luminosity. Afterwards, the influence of greenhouse gases in the daisyworld dynamics is treated establishing a comparison with the classical model. In general, these gases tend to increase the planet temperature, accelerating the death of populations and decreasing the capacity of global regulation. Thermal inertia of the planet is also of concern showing its influence in the system response. Results related to climate variability show irregular pattern that can be associated with rich responses that include chaos. Chaotic behavior of the daisyworld is investigated and is assured by the estimation of positive Lyapunov exponents. The sensitive dependence of initial condition is observed for chaotic responses of the daisyworld. Nevertheless, it is important to mention that although the system may present strong divergences due to the initial perturbation, there is a general tendency to stability in terms of global behavior, which means that the system response is basically the same during most of the time, eventually changing dramatically, but then returning to the original behavior. Besides, it should be highlighted the strong variations of system response due to parameter perturbations. The authors believe that the proposed model is useful for a qualitative description of the global warming phenomenon and further investigations should be done in order to perform a quantitative description.

Acknowledgements. The authors would like to acknowledge the support of the Brazilian Research Agencies CNPq, CAPES and FAPERJ and through the INCT-EIE (National Institute of Science and Technology - Smart Structures in Engineering) the CNPq and FAPEMIG. The Air Force Office of Scientific Research (AFOSR) is also acknowledged.

\section{REFERENCES}

[1] Ackland G.J., Wood, A.J. (2010): Emergent patterns in space and time from daisyworld: a simple evolving coupled biosphere - climate model. - Philosophical Transactions I Soc. A. 368: 161-179.

[2] Ackland, G.J., Clark, M.A., Lenton, T.M. (2003): Catastrophic desert formation in daisyworld. - Journal of Theoretical Biology 223: 39-44.

[3] Adams, B., Carr J., Lenton T.M., White A. (2003): One-dimensional daisyworld: Spatial interactions and pattern formation. - Journal of Theoretical Biology 223: 505-513.

[4] Alexiadis, A. (2007): Global warming and human activity: a model for studying the potential instability of the carbon dioxide/temperature feedback mechanism. - Ecological Modelling 203: 243-256.

[5] Charlson, R.J., Lovelock, J.E., Andreae, M.O., Warren, S.G. (1987): Oceanic phytoplankton, atmospheric sulphur, cloud albedo and climate. - Nature 326: 655-661.

[6] Cohen, J.E., Rich, A.D. (2000): Interspecific competition affects temperature stability in Daisyworld. - Tellus 52B: 980-984.

[7] De Gregorio, S., Pielke, R.A., Dalu, G.A. (1992): Feedback between a simple biosystem and the temperature of the Earth. - Journal of Nonlinear Science 2: 263-292.

[8] Foong, S.K. (2006): An accurate analytical solution of a zero-dimensional greenhouse model for global warming. - European Journal of Physics 27: 933-942. 
[9] Franca, L.F.P., Savi, M.A. (2001): Distinguishing periodic and chaotic time series obtained from an experimental nonlinear pendulum. - Nonlinear Dynamics 26(3): 253271.

[10] Franca, L.F.P., Savi, M.A. (2003): Evaluating noise sensitivity on the time series determination of Lyapunov exponents applied to nonlinear pendulum. - Shock and Vibration 10(1): 37-50.

[11] Houghton, J. (2005): Global warming. - Reports on Progress in Physics 68: 1343-1403.

[12] Jascourt, S.D., Raymond, W.H. (1992): Comments on Chaos in daisyworld by X. Zeng et al. - Tellus 44B: 243-246.

[13] Jorgensen, S.E. (1999): State-of-the-art of ecological modelling with emphasis on development of structural dynamic models. - Ecological Modelling 120(2-3): 75-96.

[14] Kantz, H. (1994): A Robust method to estimate the maximal Lyapunov exponent of a time series. - Physics Letters A 185: 77-87.

[15] Lenton, T.M., Lovelock, J.E. (2000): Daisyworld is Darwinian: constraints on adaptation are important for planetary self-regulation. - Journal of Theoretical Biology 200(206): 109-114.

[16] Lenton, T.M., Lovelock, J.E. (2001): Daisyworld revisited: quantifying biological effects on planetary self-regulation. - Tellus 53B: 288-305.

[17] Lovelock, J.E. (1983a): Gaia as seen through the atmosphere. - In: Biomineralization and biological metal accumulation (Westbroek, P., Jong, E.E. eds.) D. Reidei Publishing Company, 15-25.

[18] Lovelock, J.E. (1983b): Daisyworld - a cybernetic proof of the Gaia hypothesis. - The Co-evolution Quartely 66-72.

[19] Lovelock, J.E. (1988): The ages of Gaia - A biography of our living earth. - W.W. Norton, New York.

[20] Lovelock, J.E. (1992): A numerical model for biodiversity. - Philosophical Transactions of the Royal Society of London, Series B - Biological Sciences 338: 383-391.

[21] Nevison, C., Gupta, V., Klinger, L. (1999): Self-sustained temperature oscillations on daisyworld. - Tellus 51B: 806-814.

[22] NOAA (2011): http://www.esrl.noaa.gov.

[23] Robertson, D., Robinson, J. (1998): Darwinian daisyworld. - Journal of Theoretical Biology 195: 129-134.

[24] Salazar, J.F., Poveda, G. (2009): Role of a simplified hydrological cycle and clouds in regulating the climate - biota system of Daisyworld. - Tellus 61B: 483-497.

[25] Savi, M.A. (2005): Chaos and order in biomedical rhythms. - Journal of the Brazilian Society of Mechanical Sciences and Engineering XXVII(2): 157-169.

[26] Savi, M.A. (2006): Nonlinear dynamics and chaos. - Editora E-papers (in portuguese).

[27] Staley, M. (2002): Darwinian selection leads to Gaia. - Journal of Theoretical Biology 218: 35-46.

[28] Sugimoto, T. (2002): Darwinian evolution does not rule out the Gaia hypothesis. Journal of Theoretical Biology 218: 447-455.

[29] Viola, F.M., Paiva, S.L.D., Savi, M.A. (2010): Analysis of the Global Warming Dynamics from Temperature Time Series. - Ecological Modelling 221(16): 1964-1978.

[30] Watson, A.J., Lovelock, J.E. (1983): Biological homeostasis of global environment: the parable of daisyworld. - Tellus 35B: 284-289.

[31] WMO (2010): World Meteorological Organization, URL http://www.wmo.int, visited in march.

[32] Wood, A.J., Ackland G.J., Dyke J.G., Williams H.T.P., Lenton T.M. (2008): Daisyworld: a review. - Rev. Geophys 46. RG1001, doi:10.1029/2006RG000217.

[33] Zeng, X., Pielke, R.A., Eykholt, R. (1990): Chaos in daisyworld. - Tellus 42B: 309-318. 